\title{
Multi-Component NLS Models on Symmetric Spaces: Spectral Properties versus Representations Theory ${ }^{\star}$
}

\author{
Vladimir S. GERDJIKOV ${ }^{\dagger}$ and Georgi G. GRAHOVSKI ${ }^{\dagger}$ \\ $\dagger$ Institute for Nuclear Research and Nuclear Energy, Bulgarian Academy of Sciences, \\ 72 Tsarigradsko chaussee, 1784 Sofia, Bulgaria \\ E-mail: gerjikov@inrne.bas.bg,grah@inrne.bas.bg \\ $\ddagger$ School of Mathematical Sciences, Dublin Institute of Technology, \\ Kevin Street, Dublin 8, Ireland \\ E-mail: georgi.grahovski@dit.ie
}

Received January 20, 2010, in final form May 24, 2010; Published online June 02, 2010 doi:10.3842/SIGMA.2010.044

\begin{abstract}
The algebraic structure and the spectral properties of a special class of multicomponent NLS equations, related to the symmetric spaces of BD.I-type are analyzed. The focus of the study is on the spectral theory of the relevant Lax operators for different fundamental representations of the underlying simple Lie algebra $\mathfrak{g}$. Special attention is paid to the structure of the dressing factors in spinor representation of the orthogonal simple Lie algebras of $\mathbf{B}_{r} \simeq s o(2 r+1, \mathbb{C})$ type.
\end{abstract}

Key words: multi-component MNLS equations, reduction group, Riemann-Hilbert problem, spectral decompositions, representation theory

2010 Mathematics Subject Classification: 37K20; 35Q51; 74J30; 78A60

\section{Introduction}

The nonlinear Schrödinger equation $[49,2]$

$$
\mathrm{i} q_{t}+q_{x x}+2|q|^{2} q=0, \quad q=q(x, t)
$$

has natural multi-component generalizations. The first multi-component NLS type model with applications to physics is the so-called vector NLS equation (Manakov model) [40, 2]:

$$
i \mathbf{v}_{t}+\mathbf{v}_{x x}+2\left(\mathbf{v}^{\dagger}, \mathbf{v}\right) \mathbf{v}=0, \quad \mathbf{v}=\left(\begin{array}{c}
v_{1}(x, t) \\
\vdots \\
v_{n}(x, t)
\end{array}\right)
$$

Here $\mathbf{v}$ is an $n$-component complex-valued vector and $(\cdot, \cdot)$ is the standard scalar product. All these models appeared to be integrable by the inverse scattering method $[1,2,47,11,6,7,31,36]$.

Later on, the applications of the differential geometric and Lie algebraic methods to soliton type equations $[10,41,4,21,23,39,24,32,26,37,29,8,9]$ (for a detailed review see e.g. [31]) has lead to the discovery of a close relationship between the multi-component (matrix) NLS equations and the homogeneous and symmetric spaces [12]. It was shown that the integrable MNLS type models have Lax representation with the generalized Zakharov-Shabat system as

*This paper is a contribution to the Proceedings of the Eighth International Conference "Symmetry in Nonlinear Mathematical Physics" (June 21-27, 2009, Kyiv, Ukraine). The full collection is available at http://www.emis.de/journals/SIGMA/symmetry2009.html 
the Lax operator:

$$
L \psi(x, t, \lambda) \equiv i \frac{d \psi}{d x}+(Q(x, t)-\lambda J) \psi(x, t, \lambda)=0,
$$

where $J$ is a constant element of the Cartan subalgebra $\mathfrak{h} \subset \mathfrak{g}$ of the simple Lie algebra $\mathfrak{g}$ and $Q(x, t) \equiv[J, \widetilde{Q}(x, t)] \in \mathfrak{g} / \mathfrak{h}$. In other words $Q(x, t)$ belongs to the co-adjoint orbit $\mathcal{M}_{J}$ of $\mathfrak{g}$ passing through $J$. The Hermitian symmetric spaces, compatible with the NLS dispersion law, are labelled in Cartan classification by A.III, C.I, D.III and BD.I, see [5, 33].

In what follows we will assume that the reader is familiar with the theory of simple Lie algebras and their representations. The choice of $J$ determines the dimension of $\mathcal{M}_{J}$ which can be viewed as the phase space of the relevant nonlinear evolution equations (NLEE). It is equal to the number of roots of $\mathfrak{g}$ such that $\alpha(J) \neq 0$. Taking into account that if $\alpha$ is a root, then $-\alpha$ is also a root of $\mathfrak{g}$, so $\operatorname{dim} \mathcal{M}_{J}$ is always even.

The interpretation of the ISM as a generalized Fourier transforms and the expansion over the so-called 'squared' solutions (see [14] for regular and [20, 22] for non-regular $J$ ) are based on the spectral theory for Lax operators in the form (1.1). This allow one to study all the fundamental properties of the corresponding NLEE's: i) the description of the class of NLEE related to a given Lax operator $L(\lambda)$ and solvable by the ISM; ii) derivation of the infinite family of integrals of motion; and iii) their hierarchy of Hamiltonian structures.

Recently, it has been shown, that some of these MNLS models describe the dynamics of spinor Bose-Einstein condensates in one-dimensional approximation [34, 44]. It also allows an exact description of the dynamics and interaction of bright solitons with spin degrees of freedom [38]. Matter-wave solitons are expected to be useful in atom laser, atom interferometry and coherent atom transport (see e.g. [45] and the references therein). Furthermore, a geometric interpretation of the MNLS models describing spinor Bose-Einstein condensates are given in [12]; Darboux transformation for this special integrable case is developed in [38].

Along with multi-component NLS-type of systems, generalizations for other hierarchies has also attracted the interest of the scientific community: The scalar [46] and multi-component modified Korteweg-de Vries hierarchies over symmetric spaces [3] have been further studied in $[12,3,28]$.

It is well known that the Lax representation of the MNLS equations takes the form:

$$
[L, M]=0
$$

with conveniently chosen operator $M$, see equation (2.2) below. In other words the Lax representation is of pure Lie algebraic form and therefore the form of the MNLS is independent on the choice of the representation of the relevant Lie algebra $\mathfrak{g}$. That is why in applying the inverse scattering method (ISM) until now in solving the direct and the inverse scattering problems for $L$ (1.1) only the typical (lowest dimensional exact) representation of $\mathfrak{g}$ was used.

Our aim in this paper is to explore the spectral theory of the Lax operator $L$ (1.1) for different fundamental representations of the underlying simple Lie algebra $\mathfrak{g}$. We will see that the construction of such important for the scattering theory objects like the fundamental analytic solutions (FAS) depend crucially on the choice of the representation. This reflects on the formulation of the corresponding Riemann-Hilbert problem (RHP) and especially on the structure of the so-called dressing factors which allow one to construct the soliton solutions of the MNLS equations. In turn the dressing factors determine the structure of the singularities of the resolvent of $L$. In other words one finds the multiplicities of the discrete eigenvalues of $L$ and the structure of the corresponding eigensubspaces.

We will pay special attention to the adjoint representation, which gives the expansion over the so-called 'squared' solutions. For MNLS related to the orthogonal simple Lie algebras of $\mathbf{B}_{r}$ 
and $\mathbf{D}_{r}$ type, we will outline the spectral properties of the Lax operator in the spinor representations. Another important tool is the construction of the minimal sets of scattering data $\mathcal{T}_{i}$, $i=1,2$, each of which determines uniquely both the scattering matrix $T(\lambda)$ and the potential $Q(x)$. Our remark is that the definition of $\mathcal{T}_{i}$ used in $[14,20,25,26]$ is invariant with respect to the choice of the representation.

The paper is organized as follows: In Section 2, we give some preliminaries about MNLS type equations over symmetric spaces of BD.I-type. Here we summarize the well known facts about their Lax representations, Jost solutions and the scattering matrix $T(\lambda)$ for the typical representation, see $[14,16]$. Next we outline the construction of the FAS and the relevant RHP which they satisfy. They are constructed by using the Gauss decomposition factors of the scattering matrix $T(\lambda)$. All our constructions are applied for the class of potentials $Q(x)$ that vanish fast enough for $x \rightarrow \pm \infty$. We finish this section by brief exposition of the simplest type of dressing factors. We also introduce the minimal sets of scattering data as the minimal sets of coefficients $\mathcal{T}_{i}, i=1,2$, which determine the Gauss factors of $T(\lambda)$. These coefficient are representation independent and therefore $\mathcal{T}_{i}$ determine $T(\lambda)$ and the corresponding potential $Q(x)$ in any representation of $\mathfrak{g}$. In Section 3 we describe the spectral properties of the Lax operator in the typical representation for BD.I symmetric spaces and the effect of the dressing on the scattering data. We introduce also the kernel of the resolvent $R^{ \pm}(x, y, \lambda)$ of $L$ and use it to derive the completeness relation for the FAS in the typical representation. This relation may also be understood as the spectral decomposition of $L$. In Section 4 we describe the spectral decomposition of the Lax operator in the adjoint representation: the expansions over the 'squared' solutions and the generating (recursion) operator. Most of the results here have also been known for some time $[1,14,31]$. In particular the coefficients of $\mathcal{T}_{i}$ appear as expansion coefficients of the potential $Q(x)$ over the 'squared solutions'. This important fact allows one to treat the ISM as a generalized Fourier transform. In the next Section 5 we study the spectral properties of the same Lax operator in the spinor representation: starting with the algebraic structure of the Gauss factors for the scattering matrix, associated to $L(\lambda)$, the dressing factors, etc. Note that the FAS for BD.I-type symmetric spaces are much easier to construct in the spinor representation. One can view $L_{\mathrm{sp}}$ as Lax operator related to the algebra $s o\left(2^{r}\right)$ with additional deep reduction that picks up the spinor representation of $\mathfrak{g} \simeq s o(2 r+1)$. In the last Section 6 we extend our results to a non-fundamental representation. In order to avoid unnecessary complications we do it on the example of the 112-dimensional representation of $s o(7)$ with highest weight $\omega=3 \omega_{3}=\frac{3}{2}\left(e_{1}+e_{2}+e_{3}\right)$. The matrix realizations of both adjoint and spinor representations for $\mathbf{B}_{2} \simeq s o(5, \mathbb{C})$ and $\mathbf{B}_{7} \simeq s o(7, \mathbb{C})$ are presented in Appendices $\mathrm{A}, \mathrm{B}$ and $\mathrm{C}$.

\section{Preliminaries}

We start with some basic facts about the MNLS type models, related to BD.I symmetric spaces. Here we will present all result in the typical representation of the corresponding Lie algebra $\mathfrak{g} \simeq \mathbf{B}_{r}, \mathbf{B}_{r}$. These models admit a Lax representation in the form:

$$
\begin{aligned}
& L \psi(x, t, \lambda) \equiv i \partial_{x} \psi+(Q(x, t)-\lambda J) \psi(x, t, \lambda)=0, \\
& M \psi(x, t, \lambda) \equiv i \partial_{t} \psi+\left(V_{0}(x, t)+\lambda V_{1}(x, t)-\lambda^{2} J\right) \psi(x, t, \lambda)=0, \\
& V_{1}(x, t)=Q(x, t), \quad V_{0}(x, t)=i \operatorname{ad}_{J}^{-1} \frac{d Q}{d x}+\frac{1}{2}\left[\operatorname{ad}_{J}^{-1} Q, Q(x, t)\right] .
\end{aligned}
$$

Here, in general, $J$ is an element of the corresponding Cartan subalgebra $\mathfrak{h}$ and $Q(x, t)$ is an off-diagonal matrix, taking values in a simple Lie algebra $\mathfrak{g}$. In what follows we assume that $Q(x) \in \mathcal{M}_{J}$ is a smooth potential vanishing fast enough for $x \rightarrow \pm \infty$.

Before proceeding further on, we will fix here the notations and the normalization conditions for the Cartan-Weyl basis $\left\{h_{k}, E_{\alpha}\right\}$ of $\mathfrak{g}(r=\operatorname{rank} \mathfrak{g})$ with a root system $\Delta$. We introduce 
$h_{k} \in \mathfrak{h}, k=1, \ldots, r$ as the Cartan elements dual to the orthonormal basis $\left\{e_{k}\right\}$ in the root space $\mathbb{E}^{r}$ and the Weyl generators $E_{\alpha}, \alpha \in \Delta$. Their commutation relations are given by [5, 33]:

$$
\begin{aligned}
& {\left[h_{k}, E_{\alpha}\right]=\left(\alpha, e_{k}\right) E_{\alpha}, \quad\left[E_{\alpha}, E_{-\alpha}\right]=\frac{2}{(\alpha, \alpha)} \sum_{k=1}^{r}\left(\alpha, e_{k}\right) h_{k},} \\
& {\left[E_{\alpha}, E_{\beta}\right]= \begin{cases}N_{\alpha, \beta} E_{\alpha+\beta} & \text { for } \alpha+\beta \in \Delta, \\
0 & \text { for } \alpha+\beta \notin \Delta \cup\{0\} .\end{cases} }
\end{aligned}
$$

Here $\vec{a}=\sum_{k=1}^{r} a_{k} e_{k}$ is a $r$-dimensional vector dual to $J \in \mathfrak{h}$ and $(\cdot, \cdot)$ is the scalar product in $\mathbb{E}^{r}$. The normalization of the basis is determined by:

$$
E_{-\alpha}=E_{\alpha}^{T}, \quad\left\langle E_{-\alpha}, E_{\alpha}\right\rangle=\frac{2}{(\alpha, \alpha)}, \quad N_{-\alpha,-\beta}=-N_{\alpha, \beta},
$$

where $N_{\alpha, \beta}= \pm(p+1)$ and the integer $p \geq 0$ is such that $\alpha+s \beta \in \Delta$ for all $s=1, \ldots, p$, $\alpha+(p+1) \beta \notin \Delta$ and $\langle\cdot, \cdot\rangle$ is the Killing form of $\mathfrak{g}$, see [5,33]. The root system $\Delta$ of $\mathfrak{g}$ is invariant with respect to the group $W_{\mathfrak{g}}$ of Weyl reflections $S_{\alpha}$,

$$
S_{\alpha} \vec{y}=\vec{y}-\frac{2(\alpha, \vec{y})}{(\alpha, \alpha)} \alpha, \quad \alpha \in \Delta
$$

As it was already mentioned in the Introduction the MNLS equations correspond to Lax operator (1.1) with non-regular (constant) Cartan elements $J \in \mathfrak{h}$. If $J$ is a regular element of the Cartan subalgebra of $\mathfrak{g}$ then ad $J$ has as many different eigenvalues as is the number of the roots of the algebra and they are given by $a_{j}=\alpha_{j}(J), \alpha_{j} \in \Delta$. Such $J$ 's can be used to introduce ordering in the root system by assuming that $\alpha>0$ if $\alpha(J)>0$. In what follows we will assume that all roots for which $\alpha(J)>0$ are positive. Obviously one can consider the eigensubspaces of $\operatorname{ad}_{J}$ as grading of the algebra $\mathfrak{g}$.

In the case of symmetric spaces, the corresponding Cartan involution [33] provides a grading in $\mathfrak{g}: \mathfrak{g}=\mathfrak{g}_{0} \oplus \mathfrak{g}_{1}$, where $\mathfrak{g}_{0}$ is the subalgebra of all elements of $\mathfrak{g}$ commuting with $J$. It contains the Cartan subalgebra $\mathfrak{h}$ and the subalgebra of $\mathfrak{g} \backslash \mathfrak{h}$ spanned on those root subspaces $\mathfrak{g}_{\alpha}$, such that $\alpha(J)=0$. The set of all such roots is denoted by $\Delta_{0}$. The corresponding symmetric space is spanned by all root subspaces in $\mathfrak{g} \backslash \mathfrak{g}_{0}$. Note that one can always use a gauge transformation commuting with $J$ to remove all components of the potential $Q(x, t)$ that belong to $\mathfrak{g}_{0}$.

For symmetric spaces of BD.I type, the potential has the form:

$$
Q=\left(\begin{array}{ccc}
0 & \vec{q}^{T} & 0 \\
\vec{p} & 0 & s_{0} \vec{q} \\
0 & \vec{p}^{T} s_{0} & 0
\end{array}\right), \quad J=\operatorname{diag}(1,0, \ldots, 0,-1) .
$$

For $n=2 r+1$ the $n$-component vectors $\vec{q}$ and $\vec{p}$ have the form $\vec{q}=\left(q_{1}, \ldots, q_{r}, q_{0}, q_{\bar{r}}, \ldots, q_{\overline{1}}\right)^{T}$, $\vec{p}=\left(p_{1}, \ldots, p_{r}, p_{0}, p_{\bar{r}}, \ldots, p_{\overline{1}}\right)^{T}$, while the matrix $s_{0}=S_{0}^{(n)}$ enters in the definition of $s o(n)$ : $X \in \operatorname{so}(n), X+S_{0}^{(n)} X^{T} S_{0}^{(n)}=0$, where

$$
S_{0}^{(n)}=\sum_{s=1}^{n+1}(-1)^{s+1} E_{s, n+1-s}^{(n)} .
$$

For $n=2 r$ the $n$-component vectors $\vec{q}$ and $\vec{p}$ have the form $\vec{q}=\left(q_{1}, \ldots, q_{r}, q_{\bar{r}}, \ldots, q_{\overline{1}}\right)^{T}, \vec{p}=$ $\left(p_{1}, \ldots, p_{r}, p_{\bar{r}}, \ldots, p_{\overline{1}}\right)^{T}$ and

$$
S_{0}^{(n)}=\sum_{s=1}^{r}(-1)^{s+1}\left(E_{s, n+1-s}^{(n)}+E_{n+1-s, s}^{(n)}\right) .
$$


With this definition of orthogonality the Cartan subalgebra generators are represented by diagonal matrices. By $E_{s, p}^{(n)}$ above we mean $n \times n$ matrix whose matrix elements are $\left(E_{s, p}^{(n)}\right)_{i j}=\delta_{s i} \delta_{p j}$.

Let us comment briefly on the algebraic structure of the Lax pair, which is related to the symmetric space $S O(n+2) /(S O(n) \times S O(2))$. The element of the Cartan subalgebra $J$, which is dual to $e_{1} \in \mathbb{E}^{r}$ allows us to introduce a grading in it: $\mathfrak{g}=\mathfrak{g}_{0} \oplus \mathfrak{g}_{1}$ which satisfies:

$$
\left[X_{1}, X_{2}\right] \in \mathfrak{g}_{0}, \quad\left[X_{1}, Y_{1}\right] \in \mathfrak{g}_{1}, \quad\left[Y_{1}, Y_{2}\right] \in \mathfrak{g}_{0}
$$

for any choice of the elements $X_{1}, X_{2} \in \mathfrak{g}_{0}$ and $Y_{1}, Y_{2} \in \mathfrak{g}_{1}$. The grading splits the set of positive roots of $s o(n)$ into two subsets $\Delta^{+}=\Delta_{0}^{+} \cup \Delta_{1}^{+}$where $\Delta_{0}^{+}$contains all the positive roots of $\mathfrak{g}$ which are orthogonal to $e_{1}$, i.e. $\left(\alpha, e_{1}\right)=0$; the roots in $\beta \in \Delta_{1}^{+}$satisfy $\left(\beta, e_{1}\right)=1$. For more details see the appendix below and [33].

The Lax pair can be considered in any representation of $s o(n)$, then the potential $Q$ will take the form:

$$
Q(x, t)=\sum_{\alpha \in \Delta_{1}^{+}}\left(q_{\alpha}(x, t) E_{\alpha}+p_{\alpha}(x, t) E_{-\alpha}\right) .
$$

Next we introduce $n$-component 'vectors' formed by the Weyl generators of $s o(n+2)$ corresponding to the roots in $\Delta_{1}^{+}$:

$$
\vec{E}_{1}^{ \pm}=\left(E_{ \pm\left(e_{1}-e_{2}\right)}, \ldots, E_{ \pm\left(e_{1}-e_{r}\right)}, E_{ \pm e_{1}}, E_{ \pm\left(e_{1}+e_{r}\right)}, \ldots, E_{ \pm\left(e_{1}+e_{2}\right)}\right)
$$

for $n=2 r+1$ and

$$
\vec{E}_{1}^{ \pm}=\left(E_{ \pm\left(e_{1}-e_{2}\right)}, \ldots, E_{ \pm\left(e_{1}-e_{r}\right)}, E_{ \pm\left(e_{1}+e_{r}\right)}, \ldots, E_{ \pm\left(e_{1}+e_{2}\right)}\right),
$$

for $n=2 r$. Then the generic form of the potentials $Q(x, t)$ related to these type of symmetric spaces can be written as sum of two "scalar" products

$$
Q(x, t)=\left(\vec{q}(x, t) \cdot \vec{E}_{1}^{+}\right)+\left(\vec{p}(x, t) \cdot \vec{E}_{1}^{-}\right) .
$$

In terms of these notations the generic MNLS type equations connected to BD.I acquire the form

$$
\begin{aligned}
& i \vec{q}_{t}+\vec{q}_{x x}+2(\vec{q}, \vec{p}) \vec{q}-\left(\vec{q}, s_{0} \vec{q}\right) s_{0} \vec{p}=0, \\
& i \vec{p}_{t}-\vec{p}_{x x}-2(\vec{q}, \vec{p}) \vec{p}+\left(\vec{p}, s_{0} \vec{p}\right) s_{0} \vec{q}=0 .
\end{aligned}
$$

The Hamiltonian for the MNLS equations (2.3) with the canonical reduction $\vec{p}=\epsilon \vec{q}^{*}, \epsilon= \pm 1$ imposed, is given by:

$$
H_{\mathrm{MNLS}}=\int_{-\infty}^{\infty} d x\left(\left(\partial_{x} \vec{q}, \partial_{x} \overrightarrow{q^{*}}\right)-\epsilon\left(\vec{q}, \overrightarrow{q^{*}}\right)^{2}+\frac{\epsilon}{2}\left(\vec{q}, s_{0} \vec{q}\right)\left(\overrightarrow{q^{*}}, s_{0} \overrightarrow{q^{*}}\right)\right) .
$$

\subsection{Direct scattering problem for $L$}

The starting point for solving the direct and the inverse scattering problem (ISP) for $L$ are the so-called Jost solutions, which are defined by their asymptotics (see, e.g. [18] and the references therein):

$$
\lim _{x \rightarrow-\infty} \phi(x, t, \lambda) e^{i \lambda J x}=\mathbb{1}, \quad \lim _{x \rightarrow \infty} \psi(x, t, \lambda) e^{i \lambda J x}=\mathbb{1}
$$

and the scattering matrix $T(\lambda)$ is defined by $T(\lambda, t) \equiv \psi^{-1} \phi(x, t, \lambda)$. Here we assume that the potential $q(x, t)$ is tending to zero fast enough, when $|x| \rightarrow \infty$. The special choice of $J$ results 
in the fact that the Jost solutions and the scattering matrix take values in the corresponding orthogonal Lie group $S O(n+2)$. One can use the following block-matrix structure of $T(\lambda, t)$

$$
T(\lambda, t)=\left(\begin{array}{ccc}
m_{1}^{+} & -\vec{b}^{-T} & c_{1}^{-} \\
\vec{b}^{+} & \mathbf{T}_{22} & -s_{0} \vec{B}^{-} \\
c_{1}^{+} & \vec{B}^{+T} s_{0} & m_{1}^{-}
\end{array}\right), \quad \hat{T}(\lambda, t)=\left(\begin{array}{ccc}
m_{1}^{-} & \vec{B}^{-T} & c_{1}^{-} \\
-\vec{B}^{+} & \hat{\mathbf{T}}_{22} & s_{0} \vec{b}^{-} \\
c_{1}^{+} & -\vec{b}^{+T} s_{0} & m_{1}^{+}
\end{array}\right),
$$

where $\vec{b}^{ \pm}(\lambda, t)$ and $\vec{B}^{ \pm}(\lambda, t)$ are $n$-component vectors, $\mathbf{T}_{22}(\lambda)$ and $\hat{\mathbf{T}}_{22}(\lambda)$ are $n \times n$ block matrices, and $m_{1}^{ \pm}(\lambda), c_{1}^{ \pm}(\lambda)$ are scalar functions. Here and below by 'hat' we will denote taking the inverse, i.e. $\hat{T}(\lambda, t)=T^{-1}(\lambda)$. Such parametrization is compatible with the generalized Gauss decompositions [33] of $T(\lambda, t)$.

With this notations we introduce the generalized Gauss factors of $T(\lambda)$ as follows:

$$
\begin{aligned}
& T(\lambda, t)=T_{J}^{-} D_{J}^{+} \hat{S}_{J}^{+}=T_{J}^{+} D_{J}^{-} \hat{S}_{J}^{-}, \\
& T_{J}^{-}=e^{\left(\vec{\rho}^{+}, \vec{E}^{-}\right)}=\left(\begin{array}{ccc}
1 & 0 & 0 \\
\vec{\rho}^{+} & \mathbb{1} & 0 \\
c_{1}^{\prime \prime},+ & \vec{\rho}^{+, T} & s_{0}
\end{array}\right), \quad T_{J}^{+}=e^{\left(-\vec{\rho}^{-}, \vec{E}^{+}\right)}=\left(\begin{array}{ccc}
1 & -\vec{\rho}^{-, T} & c_{1}^{\prime \prime},- \\
0 & \mathbb{1} & -s_{0} \vec{\rho}^{-} \\
0 & 0 & 1
\end{array}\right), \\
& S_{J}^{+}=e^{\left(\vec{\tau}^{+}, \vec{E}^{+}\right)}=\left(\begin{array}{ccc}
1 & \vec{\tau}^{+, T} & c_{1}^{\prime}- \\
0 & \mathbb{1} & s_{0} \vec{\tau}^{+} \\
0 & 0 & 1
\end{array}\right), \quad S_{J}^{-}=e^{\left(-\vec{\tau}^{-}, \vec{E}^{-}\right)}=\left(\begin{array}{ccc}
1 & 0 & 0 \\
-\vec{\tau}^{-} & \mathbb{1} & 0 \\
c_{1}^{\prime,+} & -\vec{\tau}^{-, T} s_{0} & 1
\end{array}\right) \text {, } \\
& D_{J}^{+}=\left(\begin{array}{ccc}
m_{1}^{+} & 0 & 0 \\
0 & \boldsymbol{m}_{2}^{+} & 0 \\
0 & 0 & 1 / m_{1}^{+}
\end{array}\right), \quad D_{J}^{-}=\left(\begin{array}{ccc}
1 / m_{1}^{-} & 0 & 0 \\
0 & \boldsymbol{m}_{2}^{-} & 0 \\
0 & 0 & m_{1}^{-}
\end{array}\right) \text {, } \\
& c_{1}^{\prime \prime}{ }^{\prime \prime}=\frac{1}{2}\left(\vec{\rho}^{ \pm, T} s_{0} \vec{\rho}^{ \pm}\right), \quad c_{1}^{\prime}{ }^{\prime \pm}=\frac{1}{2}\left(\vec{\tau}^{\mp, T} s_{0} \vec{\tau}^{\mp}\right),
\end{aligned}
$$

where

$$
\begin{aligned}
& c_{1}^{-}=\frac{m_{1}^{-}}{2}\left(\vec{\rho}, T s_{0} \vec{\rho}\right)=\frac{m_{1}^{+}}{2}\left(\vec{\tau}^{+, T} s_{0} \vec{\tau}^{+}\right), \quad c_{1}^{+}=\frac{m_{1}^{+}}{2}\left(\vec{\rho}^{+, T} s_{0} \vec{\rho}^{+}\right)=\frac{m_{1}^{-}}{2}\left(\vec{\tau}^{-, T} s_{0} \vec{\tau}^{-}\right), \\
& \vec{\rho}=\frac{\vec{B}^{-}}{m_{1}^{-}}, \quad \vec{\tau}^{-}=\frac{\vec{B}^{+}}{m_{1}^{-}}, \quad \vec{\rho}^{+}=\frac{\vec{b}^{+}}{m_{1}^{+}}, \quad \vec{\tau}^{+}=\frac{\vec{b}^{-}}{m_{1}^{+}}, \\
& \boldsymbol{m}_{2}^{+}=\mathbf{T}_{22}+\frac{\vec{b}^{+} \vec{b}^{-T}}{m_{1}^{+}}, \quad \boldsymbol{m}_{2}^{-}=\mathbf{T}_{22}+\frac{s_{0} \vec{b}^{-} \vec{b}^{+T} s_{0}}{m_{1}^{-}} .
\end{aligned}
$$

These notations satisfy a number of relations which ensure that both $T(\lambda)$ and its inverse $\hat{T}(\lambda)$ belong to the corresponding orthogonal group $S O(n+2)$ and that $T(\lambda) \hat{T}(\lambda)=\mathbb{1}$. Some of them take the form:

$$
\begin{array}{ll}
m_{1}^{+} m_{1}^{-}+\left(\vec{b}^{-}, \vec{B}^{+}\right)+c_{1}^{+} c_{1}^{-}=1, & \vec{b}^{+} \vec{B}^{-T}+\mathbf{T}_{22} s_{0} \mathbf{T}_{22}^{T} s_{0}+s_{0} \vec{B}^{-} \vec{b}^{+T} s_{0}=\mathbb{1}, \\
2 m_{1}^{+} c_{1}^{-}-\vec{b}^{-, T} s_{0} \vec{b}^{-}=0, & 2 m_{1}^{-} c_{1}^{+}-\vec{B}^{+, T} s_{0} \vec{B}^{+}=0, \\
m_{1}^{-} \vec{b}^{+}-\mathbf{T}_{22} \vec{B}^{+}-s_{0} \vec{B}^{-} c_{1}^{+}=0, & m_{1}^{+} \vec{B}^{-}-\hat{\mathbf{T}}_{22}^{T} \vec{b}^{-}-s_{0} \vec{b}^{+} c_{1}^{-}=0 .
\end{array}
$$

Important tools for reducing the ISP to a Riemann-Hilbert problem (RHP) are the fundamental analytic solution (FAS) $\chi^{ \pm}(x, t, \lambda)$. Their construction is based on the generalized Gauss decomposition of $T(\lambda, t)$, see $[47,14,16]$ :

$$
\chi^{ \pm}(x, t, \lambda)=\phi(x, t, \lambda) S_{J}^{ \pm}(t, \lambda)=\psi(x, t, \lambda) T_{J}^{\mp}(t, \lambda) D_{J}^{ \pm}(\lambda) .
$$

More precisely, this construction ensures that $\xi^{ \pm}(x, \lambda)=\chi^{ \pm}(x, \lambda) e^{i \lambda J x}$ are analytic functions of $\lambda$ for $\lambda \in \mathbb{C}_{ \pm}$. Here $S_{J}^{ \pm}, T_{J}^{ \pm}$upper- and lower-block-triangular matrices, while $D_{J}^{ \pm}(\lambda)$ are 
block-diagonal matrices with the same block structure as $T(\lambda, t)$ above. Skipping the details, we give here the explicit expressions of the Gauss factors in terms of the matrix elements of $T(\lambda, t)$

$$
S_{J}^{ \pm}(t, \lambda)=\exp \left( \pm\left(\vec{\tau}^{ \pm}(\lambda, t) \cdot \vec{E}_{1}^{ \pm}\right)\right), \quad T_{J}^{ \pm}(t, \lambda)=\exp \left(\mp\left(\vec{\rho}^{\mp}(\lambda, t) \cdot \vec{E}_{1}^{ \pm}\right)\right),
$$

where

$$
\vec{\tau}^{+}(\lambda, t)=\frac{\vec{b}^{-}}{m_{1}^{+}}, \quad \vec{\tau}^{-}(\lambda, t)=\frac{\vec{B}^{+}}{m_{1}^{-}}, \quad \vec{\rho}^{+}(\lambda, t)=\frac{\vec{b}^{+}}{m_{1}^{+}}, \quad \vec{\rho}(\lambda, t)=\frac{\vec{B}^{-}}{m_{1}^{-}},
$$

and

$$
\begin{array}{ll}
\mathbf{T}_{22}=\boldsymbol{m}_{2}^{+}-\frac{\vec{b}^{+} \vec{b}^{-T}}{2 m_{1}^{+}}, & \mathbf{T}_{22}=\boldsymbol{m}_{2}^{-}-\frac{s_{0} \vec{b}^{-} \vec{b}^{+T} s_{0}}{2 m_{1}^{-}}, \\
\hat{\mathbf{T}}_{22}=\hat{\boldsymbol{m}}_{2}^{+}-\frac{s_{0} \vec{b}^{-} \vec{b}^{+T} s_{0}}{2 m_{1}^{+}}, & \hat{\mathbf{T}}_{22}=\hat{\boldsymbol{m}}_{2}^{-}-\frac{\vec{B}^{+} \vec{B}^{-T}}{2 m_{1}^{-}}
\end{array}
$$

The two analyticity regions $\mathbb{C}_{+}$and $\mathbb{C}_{-}$are separated by the real line. The continuous spectrum of $L$ fills in the real line and has multiplicity 2, see Section 3.3 below.

If $Q(x, t)$ evolves according to $(2.3)$ then the scattering matrix and its elements satisfy the following linear evolution equations

$$
i \frac{d \vec{b}^{ \pm}}{d t} \pm \lambda^{2} \vec{b}^{ \pm}(t, \lambda)=0, \quad i \frac{d \vec{B}^{ \pm}}{d t} \pm \lambda^{2} \vec{B}^{ \pm}(t, \lambda)=0, \quad i \frac{d m_{1}^{ \pm}}{d t}=0, \quad i \frac{d \boldsymbol{m}_{2}^{ \pm}}{d t}=0
$$

so the block-diagonal matrices $D^{ \pm}(\lambda)$ can be considered as generating functionals of the integrals of motion. It is well known $[10,4,12,14]$ that generic nonlinear evolution equations related to a simple Lie algebra $\mathfrak{g}$ of rank $r$ possess $r$ series of integrals of motion in involution; thus for them one can prove complete integrability [4]. In our case we can consider as generating functionals of integrals of motion all $(2 r-1)^{2}$ matrix elements of $\boldsymbol{m}_{2}^{ \pm}(\lambda)$, as well as $m_{1}^{ \pm}(\lambda)$ for $\lambda \in \mathbb{C}_{ \pm}$. However they can not be all in involution. Such situation is characteristic for the superintegrable models. It is due to the degeneracy of the dispersion law of (2.3). We remind that $D_{J}^{ \pm}(\lambda)$ allow analytic extension for $\lambda \in \mathbb{C}_{ \pm}$and that their zeroes and poles determine the discrete eigenvalues of $L$.

\subsection{Riemann-Hilbert problem and minimal set of scattering data for $L$}

The FAS for real $\lambda$ are linearly related

$$
\chi^{+}(x, t, \lambda)=\chi^{-}(x, t, \lambda) G_{0, J}(\lambda, t), \quad G_{0, J}(\lambda, t)=\hat{S}_{J}^{-}(\lambda, t) S_{J}^{+}(\lambda, t) .
$$

One can rewrite equation (2.7) in an equivalent form for the $\operatorname{FAS} \xi^{ \pm}(x, t, \lambda)=\chi^{ \pm}(x, t, \lambda) e^{i \lambda J x}$ which satisfy the equation:

$$
i \frac{d \xi^{ \pm}}{d x}+Q(x) \xi^{ \pm}(x, \lambda)-\lambda\left[J, \xi^{ \pm}(x, \lambda)\right]=0, \quad \lim _{\lambda \rightarrow \infty} \xi^{ \pm}(x, t, \lambda)=\mathbb{1} .
$$

Then these FAS satisfy

$$
\xi^{+}(x, t, \lambda)=\xi^{-}(x, t, \lambda) G_{J}(x, \lambda, t), \quad G_{J}(x, \lambda, t)=e^{-i \lambda J x} G_{0, J}^{-}(\lambda, t) e^{i \lambda J x} .
$$

Obviously the sewing function $G_{J}(x, \lambda, t)$ is uniquely determined by the Gauss factors $S_{J}^{ \pm}(\lambda, t)$. Equation (2.9) is a Riemann-Hilbert problem (RHP) in multiplicative form. Since the Lax operator has no discrete eigenvalues, $\operatorname{det} \xi^{ \pm}(x, \lambda)$ have no zeroes for $\lambda \in \mathbb{C}_{ \pm}$and $\xi^{ \pm}(x, \lambda)$ are 
regular solutions of the RHP. As it is well known the regular solution $\xi^{ \pm}(x, \lambda)$ of the RHP is uniquely determined.

Given the solutions $\xi^{ \pm}(x, t, \lambda)$ one recovers $Q(x, t)$ via the formula

$$
Q(x, t)=\lim _{\lambda \rightarrow \infty} \lambda\left(J-\xi^{ \pm} J \widehat{\xi}^{ \pm}(x, t, \lambda)\right)=\left[J, \xi_{1}(x)\right]
$$

which is obtained from equation (2.8) taking the limit $\lambda \rightarrow \infty$. By $\xi_{1}(x)$ above we have denoted $\xi_{1}(x)=\lim _{\lambda \rightarrow \infty} \lambda(\xi(x, \lambda)-\mathbb{1})$.

If the potential $Q(x, t)$ is such that the Lax operator $L$ has no discrete eigenvalues, then the minimal set of scattering data is given by one of the sets $\mathfrak{T}_{i}, i=1,2$

$$
\begin{aligned}
& \mathfrak{T}_{1} \equiv\left\{\rho_{\alpha}^{+}(\lambda, t), \rho_{\alpha}^{-}(\lambda, t), \alpha \in \Delta_{1}^{+}, \lambda \in \mathbb{R}\right\}, \\
& \mathfrak{T}_{2} \equiv\left\{\tau_{\alpha}^{+}(\lambda, t), \tau_{\alpha}^{-}(\lambda, t), \quad \alpha \in \Delta_{1}^{+}, \lambda \in \mathbb{R}\right\} .
\end{aligned}
$$

Any of these sets determines uniquely the scattering matrix $T(\lambda, t)$ and the corresponding potential $Q(x, t)$. For more details, we refer to $[18,11,47]$ and references therein.

Most of the known examples of MNLS on symmetric spaces are obtained after imposing the reduction:

$$
\begin{aligned}
& Q(x, t)=K_{0}^{-1} Q^{\dagger}(x, t) K_{0}, \quad K_{0}=\exp \left(\frac{\pi i}{2} \sum_{j=1}^{r}\left(3+\epsilon_{j}\right) H_{j}\right), \\
& p_{k}=\tilde{K}_{0} q_{k}^{*}, \quad \tilde{K}_{0}=\operatorname{diag}\left(K_{0,22}, \ldots, K_{0, n+1, n+1}\right)
\end{aligned}
$$

or in components $p_{k}=\epsilon_{1} \epsilon_{k} q_{k}^{*}$. As a consequence the corresponding MNLS takes the form:

$$
i \vec{q}_{t}+\vec{q}_{x x}+2\left(\vec{q}, \tilde{K}_{0} \vec{q}^{*}\right) \vec{q}-\left(\vec{q}, s_{0} \vec{q}\right) s_{0} \tilde{K}_{0} \vec{q}^{*}=0 .
$$

The scattering data are restricted by $\vec{\rho}^{-}(\lambda, t)=\tilde{K}_{0} \vec{\rho}^{+, *}(\lambda, t)$ and $\vec{\tau}^{-}(\lambda, t)=\tilde{K}_{0} \vec{\tau}^{+, *}(\lambda, t)$.

If all $\epsilon_{j}=1$ then the reduction becomes the "canonical" one: $Q(x, t)=Q^{\dagger}(x, t)$ and $\vec{\rho}-(\lambda, t)=$ $\vec{\rho}^{+, *}(\lambda, t)$ and $\vec{\tau}^{-}(\lambda, t)=\vec{\tau}^{+, *}(\lambda, t)$.

\subsection{Dressing factors and soliton solutions}

The main goal of the dressing method $[50,23,35,24,32,15]$ is, starting from a known solutions $\chi_{0}^{ \pm}(x, t, \lambda)$ of $L_{0}(\lambda)$ with potential $Q_{(0)}(x, t)$ to construct new singular solutions $\chi_{1}^{ \pm}(x, t, \lambda)$ of $L$ with a potential $Q_{(1)}(x, t)$ with two additional singularities located at prescribed positions $\lambda_{1}^{ \pm}$; the reduction $\vec{p}=\vec{q}^{*}$ ensures that $\lambda_{1}^{-}=\left(\lambda_{1}^{+}\right)^{*}$. It is related to the regular one by a dressing factor $u(x, t, \lambda)$

$$
\chi_{1}^{ \pm}(x, t, \lambda)=u(x, \lambda) \chi_{0}^{ \pm}(x, t, \lambda) u_{-}^{-1}(\lambda), \quad u_{-}(\lambda)=\lim _{x \rightarrow-\infty} u(x, \lambda) .
$$

Note that $u_{-}(\lambda)$ is a block-diagonal matrix. The dressing factor $u(x, \lambda)$ must satisfy the equation

$$
i \partial_{x} u+Q_{(1)}(x) u-u Q_{(0)}(x)-\lambda[J, u(x, \lambda)]=0,
$$

and the normalization condition $\lim _{\lambda \rightarrow \infty} u(x, \lambda)=\mathbb{1}$. The construction of $u(x, \lambda) \in S O(n+2)$ is based on an appropriate anzatz specifying explicitly the form of its $\lambda$-dependence (see [48, 32] and the references therein). Here we will consider a special choice of dressing factors:

$$
u(x, \lambda)=\mathbb{1}+(c(\lambda)-1) P(x)+\left(\frac{1}{c(\lambda)}-1\right) \bar{P}(x),
$$




$$
\bar{P}=S_{0}^{-1} P^{T} S_{0}, \quad c(\lambda)=\frac{\lambda-\lambda_{1}^{+}}{\lambda-\lambda_{1}^{-}}
$$

where $P(x)$ and $\bar{P}(x)$ are mutually orthogonal projectors with rank 1 . More specifically we have

$$
P(x, t)=\frac{|n(x, t)\rangle\langle m(x, t)|}{\langle n(x, t) \mid m(x, t)\rangle},
$$

where $\langle m(x, t)|=\left\langle m_{0}\right|\left(\chi_{0}^{-}\left(x, \lambda_{1}^{-}\right)\right)^{-1}$ and $|n(x, t)\rangle=\chi_{0}^{+}\left(x, \lambda_{1}^{+}\right)\left|n_{0}\right\rangle ;\left\langle m_{0}\right|$ and $\left|n_{0}\right\rangle$ are (constant) polarization vectors [23]. Taking the limit $\lambda \rightarrow \infty$ in equation (2.12) we get that

$$
Q_{(1)}(x, t)-Q_{(0)}(x, t)=\left(\lambda_{1}^{-}-\lambda_{1}^{+}\right)[J, P(x, t)-\bar{P}(x, t)] .
$$

Below we assume that $Q_{(0)}=0$ and impose $\mathbb{Z}_{2}$ reduction condition

$$
K Q K^{-1}=Q, \quad K=\operatorname{diag}\left(\epsilon_{1}, \epsilon_{2}, \ldots, \epsilon_{r-1}, 1, \epsilon_{r}, \epsilon_{r-1}, \ldots, \epsilon_{2}, \epsilon_{1}\right) .
$$

This in its turn leads to $\lambda_{1}^{ \pm}=\mu \pm i \nu$ and $\left|m_{a}\right\rangle=K_{a}\left|n_{a}\right\rangle^{*}, a=1, \ldots, n+2$. The polarization vectors $\langle m(x, t)|$ and $|n(x, t)\rangle$ are parameterised as follows:

$$
\langle m(x, t)|=\left(m_{1}(x, t), m_{2}(x, t), \ldots, m_{r}(x, t), 0, m_{r}(x, t), \ldots, m_{2}(x, t), m_{1}(x, t)\right)
$$

and

$$
|n(x, t)\rangle=\left(n_{1}(x, t), n_{2}(x, t), \ldots, n_{r}(x, t), 0, n_{r}(x, t), \ldots, n_{2}(x, t), n_{1}(x, t)\right)^{T} .
$$

As a result one gets:

$$
q_{k}^{(1 \mathrm{~s})}(x, t)=-2 i \nu\left(P_{1 k}(x, t)+(-1)^{k} P_{\bar{k}, n+2}(x, t)\right),
$$

where $\bar{k}=n+3-k$. For more details on the soliton solutions satisfying the standard reduction $Q(x, t)=Q^{\dagger}(x, t)$ see $[29,35,37,24,32,22]$.

The effect of the dressing on the scattering data (2.5) is as follows:

$$
\begin{array}{ll}
\vec{\rho}_{1}^{+}=\frac{\vec{b}^{+}}{m_{1}^{+}}=\frac{1}{c(\lambda)} \vec{\rho}_{0}^{+}, & \overrightarrow{\rho_{1}}=\frac{\vec{B}^{-}}{m_{1}^{-}}=c(\lambda) \vec{\rho}_{0}^{-} ; \\
\vec{\tau}_{1}^{+}=\frac{\vec{b}^{-}}{m_{1}^{+}}=\frac{1}{c(\lambda)} \vec{\tau}_{0}^{+}, & \vec{\tau}_{1}^{-}=\frac{\vec{B}^{+}}{m_{1}^{-}}=c(\lambda) \vec{\tau}_{0}^{-} .
\end{array}
$$

Applying $N$ times the dressing method, one gets a Lax operator with $N$ pairs of prescribed discrete eigenvalues $\lambda_{j}^{ \pm}, j=1, \ldots, N$. The minimal sets of scattering data for the "dressed" Lax operator contains in addition the discrete eigenvalues $\lambda_{j}^{ \pm}, j=1, \ldots, N$ and the corresponding reflection/transmission coefficient at these points:

$$
\begin{aligned}
& \mathfrak{T}_{1}^{\prime} \equiv\left\{\rho_{\alpha}^{+}(\lambda, t), \rho_{\alpha}^{-}(\lambda, t), \rho_{\alpha, j}^{+}(t), \rho_{\alpha, j}^{+}(t), \lambda_{j}^{ \pm}, \alpha \in \Delta_{1}^{+}, \lambda \in \mathbb{R}, j=1, \ldots, N\right\}, \\
& \mathfrak{T}_{2}^{\prime} \equiv\left\{\tau_{\alpha}^{+}(\lambda, t), \tau_{\alpha}^{-}(\lambda, t), \tau_{\alpha, j}^{+}(t), \tau_{\alpha, j}^{+}(t), \lambda_{j}^{ \pm}, \alpha \in \Delta_{1}^{+}, \lambda \in \mathbb{R}, j=1, \ldots, N\right\} .
\end{aligned}
$$

\section{Resolvent and spectral decompositions in the typical representation of $\mathfrak{g} \simeq B_{r}$}

Here we first formulate the interrelation between the 'naked' and dressed FAS. We will use these relations to determine the order of pole singularities of the resolvent which of course will influence the contribution of the discrete spectrum to the completeness relation.

We first start with the simplest and more general case of the generalized Zakharov-Shabat system in which all eigenvalues of $J$ are different. Then we discuss the additional construction necessary to treat cases when $J$ has vanishing and/or equal eigenvalues. 


\subsection{The effect of dressing on the scattering data}

Let us first determine the effect of dressing on the Jost solutions with the simplest dressing factor $u_{1}(x, \lambda)$. In what follows below we denote the Jost solutions corresponding to the regular solutions of RHP by $\psi_{0}(x, \lambda)$ and the dressed one by $\psi_{1}(x, \lambda)$. In order to preserve the definition in equation (2.4) we put:

$$
\psi_{1}(x, \lambda)=u_{1}(x, \lambda) \psi_{0}(x, \lambda) \hat{u}_{1,+}(\lambda), \quad \phi_{1}(x, \lambda)=u_{1}(x, \lambda) \phi_{0}(x, \lambda) \hat{u}_{1,-}(\lambda),
$$

where $u_{1, \pm}(x, \lambda)=\lim _{x \rightarrow \pm \infty} u_{1}(x, \lambda)$. We will also use the fact that $u_{1, \pm}(\lambda)$ are $x$-independent elements belonging to the Cartan subgroup of $\mathfrak{g}$. For the typical representation of $s o(2 r+1)$ and for the case in which only two singularities $\lambda_{1}^{ \pm}$are added we have:

$$
\begin{aligned}
& u(x, \lambda)=\exp \left(\ln c_{1}(\lambda)\left(P_{1}-\bar{P}_{1}\right)\right), \\
& u_{1,+}(\lambda)=\mathbb{1}+\left(c_{1}(\lambda)-1\right) E_{11}+\left(\frac{1}{c_{1}(\lambda)}-1\right) E_{n+2, n+2}, \\
& u_{1,-}(\lambda)=\mathbb{1}+\left(c_{1}(\lambda)-1\right) E_{n+2, n+2}+\left(\frac{1}{c_{1}(\lambda)}-1\right) E_{11}, \\
& u_{1, \pm}(\lambda)=\exp \left( \pm \ln c_{1}(\lambda) J\right) .
\end{aligned}
$$

Then from equation (2.6) we get:

$$
\chi_{1}^{ \pm}(x, \lambda)=u_{1}(x, \lambda) \chi_{0}^{ \pm}(x, \lambda) \hat{u}_{1,-}(\lambda) .
$$

As a consequence we find that

$$
\begin{array}{ll}
T_{1}(\lambda)=u_{1,+}(\lambda) T_{0}(\lambda) \hat{u}_{1,-}(\lambda), & D_{1}^{ \pm}(\lambda)=u_{1,+}(\lambda) D_{0}(\lambda) \hat{u}_{1,-}(\lambda), \\
S_{1}^{ \pm}(\lambda)=u_{1,-}(\lambda) S_{0}(\lambda) \hat{u}_{1,-}(\lambda), & T_{1}^{ \pm}(\lambda)=u_{1,+}(\lambda) T_{0}(\lambda) \hat{u}_{1,+}(\lambda) .
\end{array}
$$

One can repeat the dressing procedure $N$ times by using the dressing factor:

$$
u(x, \lambda)=u_{N}(x, \lambda) u_{N-1}(x, \lambda) \cdots u_{1}(x, \lambda) .
$$

Note that the projector $P_{k}$ of the $k$-th dressing factor has the form of (2.14) but the $x$-dependence of the polarization vectors is determined by the $k-1$ dressed FAS:

$$
\begin{aligned}
& \chi_{k}^{ \pm}(x, \lambda)=u_{k}(x, \lambda) u_{k-1}(x, \lambda) \cdots u_{1}(x, \lambda) \chi_{0}^{ \pm}(x, \lambda) \hat{u}_{1,-}(\lambda) \cdots \hat{u}_{k-1,-}(\lambda) \hat{u}_{k,-}(\lambda), \\
& u_{k}(x, \lambda)=\mathbb{1}+\left(c_{k}(\lambda)-1\right) P_{k}(x)+\left(\frac{1}{c_{k}(\lambda)}-1\right) \bar{P}_{k}(x), \quad \bar{P}_{k}=S_{0}^{-1} P_{k}^{T} S_{0}, \\
& c_{k}(\lambda)=\frac{\lambda-\lambda_{k}^{+}}{\lambda_{k}-\lambda_{1}^{-}}, \quad P_{k}(x, t)=\frac{\left|n_{k}(x, t)\right\rangle\left\langle m_{k}(x, t)\right|}{\left\langle n_{k}(x, t) \mid m_{k}(x, t)\right\rangle}, \\
& \left\langle m_{k}(x, t)\left|=\left\langle m_{0, k}\left|\left(\chi_{k-1}^{-}\left(x, \lambda_{k}^{-}\right)\right)^{-1}, \quad\right| n_{k}(x, t)\right\rangle=\chi_{k-1}^{+}\left(x, \lambda_{k}^{+}\right)\right| n_{0, k}\right\rangle,
\end{aligned}
$$

and $\left\langle m_{0, k}\right|$ and $\left|n_{0, k}\right\rangle$ are (constant) polarization vectors

Using equation (3.4) and assuming that all $\lambda_{j} \in \mathbb{C}_{ \pm}$are different we can treat the general case of RHP with $2 N$ singular points. The corresponding relations between the 'naked' and dressed FAS are:

$$
\begin{array}{ll}
T(\lambda)=u_{-}(\lambda) T_{0}(\lambda) \hat{u}_{-}(\lambda), & D^{ \pm}(\lambda)=u_{+}(\lambda) D_{0}(\lambda) \hat{u}_{-}(\lambda), \\
S^{ \pm}(\lambda)=u_{-}(\lambda) S_{0}(\lambda) \hat{u}_{-}(\lambda), & T^{ \pm}(\lambda)=u_{+}(\lambda) T_{0}(\lambda) \hat{u}_{+}(\lambda),
\end{array}
$$


where

$$
\begin{aligned}
& u_{+}(\lambda)=\mathbb{1}+(c(\lambda)-1) E_{1,1}+\left(\frac{1}{c(\lambda)}-1\right) E_{n+2, n+2}, \\
& u_{-}(\lambda)=\mathbb{1}+(c(\lambda)-1) E_{n+2, n+2}+\left(\frac{1}{c(\lambda)}-1\right) E_{1,1}, \\
& u_{ \pm}(\lambda)=\exp ( \pm \ln c(\lambda) J), \quad c(\lambda)=\prod_{k=1}^{N} c_{k}(\lambda) .
\end{aligned}
$$

In components equations (3.6) give:

$$
\begin{array}{lll}
m_{1}^{+}(\lambda)=m_{1,0}^{+}(\lambda) c^{2}(\lambda), & m_{1}^{-}(\lambda)=\frac{m_{1,0}^{-}(\lambda)}{c^{2}(\lambda)}, \\
\rho_{1}^{+}(\lambda)=\frac{\rho_{0}^{+}(\lambda)}{c(\lambda)}, & \rho_{1}^{-}(\lambda)=c(\lambda) \rho_{0}^{-}(\lambda), \\
\tau_{1}^{+}(\lambda)=\frac{\tau_{0}^{+}(\lambda)}{c(\lambda)}, & \tau_{1}^{-}(\lambda)=c(\lambda) \tau_{0}^{-}(\lambda),
\end{array}
$$

and $\boldsymbol{m}_{2}^{ \pm}(\lambda)=\boldsymbol{m}_{2,0}^{ \pm}(\lambda)$.

In what follows we will need the residues of $u(x, \lambda) \chi_{0}^{ \pm}(x, \lambda)$ and its inverse $\hat{\chi}_{0}^{ \pm}(x, \lambda) \hat{u}(x, \lambda)$ at $\lambda=\lambda_{k}^{ \pm}$respectively. From equations (3.4) and (3.5) we get:

$$
\begin{aligned}
& u(x, \lambda) \chi_{0}^{+}(x, \lambda) \simeq \frac{\left(\lambda_{k}^{-}-\lambda_{k}^{+}\right) \chi^{+,(k)}(x)}{\lambda-\lambda_{k}^{+}}+\dot{\chi}^{+,(k)}(x)+\mathcal{O}\left(\lambda-\lambda_{k}^{+}\right), \\
& u(x, \lambda) \chi_{0}^{-}(x, \lambda) \simeq \frac{\left(\lambda_{k}^{+}-\lambda_{k}^{-}\right) \chi^{-,(k)}(x)}{\lambda-\lambda_{k}^{-}}+\dot{\chi}^{-,(k)}(x)+\mathcal{O}\left(\lambda-\lambda_{k}^{-}\right), \\
& \hat{\chi}_{0}^{+}(x, \lambda) \hat{u}(x, \lambda) \simeq \frac{\left(\lambda_{k}^{-}-\lambda_{k}^{+}\right) \hat{\chi}^{+,(k)}(x)}{\lambda-\lambda_{k}^{+}}+\hat{\dot{\chi}}^{+,(k)}(x)+\mathcal{O}\left(\lambda-\lambda_{k}^{+}\right), \\
& \hat{\chi}_{0}^{-}(x, \lambda) \hat{u}(x, \lambda) \simeq \frac{\left(\lambda_{k}^{+}-\lambda_{k}^{-}\right) \hat{\chi}^{-,(k)}(x)}{\lambda-\lambda_{k}^{-}}+\hat{\dot{\chi}}^{-,(k)}(x)+\mathcal{O}\left(\lambda-\lambda_{k}^{-}\right),
\end{aligned}
$$

where

$$
\begin{aligned}
& \chi^{+,(k)}(x)=u_{N}\left(x, \lambda_{k}^{+}\right) \cdots u_{k+1}\left(x, \lambda_{k}^{+}\right) \bar{P}_{k} \chi_{(k-1)}^{+}\left(x, \lambda_{k}^{+}\right), \\
& \chi^{-,(k)}(x)=u_{N}\left(x, \lambda_{k}^{-}\right) \cdots u_{k+1}\left(x, \lambda_{k}^{-}\right) P_{k} \chi_{(k-1)}^{-}\left(x, \lambda_{k}^{-}\right), \\
& \hat{\chi}^{+,(k)}(x)=\hat{\chi}_{(k-1)}^{+}\left(x, \lambda_{k}^{+}\right) \hat{u}_{k+1}\left(x, \lambda_{k}^{+}\right) \cdots \hat{u}_{N}\left(x, \lambda_{k}^{+}\right) P_{k}, \\
& \hat{\chi}^{-,(k)}(x)=\hat{\chi}_{(k-1)}^{-}\left(x, \lambda_{k}^{-}\right) \hat{u}_{k+1}\left(x, \lambda_{k}^{-}\right) \cdots \hat{u}_{N}\left(x, \lambda_{k}^{-}\right) \bar{P}_{k} .
\end{aligned}
$$

These results will be used below to find the residues of the resolvent at $\lambda=\lambda_{k}^{ \pm}$.

\subsection{Spectral decompositions for the generalized Zakharov-Shabat system: $\operatorname{sl}(n)$-case}

The FAS are the basic tool in constructing the spectral theory of the corresponding Lax operator. For the generic Lax operators related to the $\operatorname{sl}(n)$ algebras:

$$
L_{\text {gen }} \equiv i \frac{\partial \chi_{\text {gen }}}{\partial x}+\left(Q_{\text {gen }}(x)-\lambda J_{\text {gen }}\right) \chi_{\text {gen }}(x, \lambda)=0, \quad\left(Q_{\text {gen }}\right)_{j j}(x)=0,
$$


this theory is well developed, see $[47,19,14,17]$. In the generic case all eigenvalues of $J_{\text {gen }}=$ $\operatorname{diag}\left(J_{1}, J_{2}, \ldots, J_{n+2}\right)$ are different and non-vanishing:

$$
J_{1}>J_{2}>\cdots>J_{k}>0>J_{k+1}>\cdots>J_{n+2}, \quad \operatorname{tr} J_{\text {gen }}=0 .
$$

The Jost solutions $\psi_{\text {gen }}(x, \lambda), \phi_{\text {gen }}(x, \lambda)$, the scattering matrix $T_{\text {gen }}(\lambda)$ and the FAS $\chi_{\text {gen }}^{ \pm}(x, \lambda)$ are introduced by $[43,47]$ (see also $[42,14,17,18,19,22]$ ):

$$
\begin{array}{ll}
\lim _{x \rightarrow-\infty} \phi_{\text {gen }}(x, \lambda) e^{i J_{\operatorname{gen}} x \lambda}=\mathbb{1}, & \lim _{x \rightarrow \infty} \psi_{\text {gen }}(x, \lambda) e^{i J_{\text {gen }} x \lambda}=\mathbb{1}, \\
T_{\text {gen }}(\lambda)=\psi_{\text {gen }}^{-1}(x, \lambda) \phi_{\text {gen }}(x, \lambda), & \\
\chi_{\text {gen }}^{ \pm}(x, \lambda)=\phi_{\text {gen }}(x, \lambda) S_{\text {gen }}^{ \pm}(\lambda), & \chi_{\text {gen }}^{ \pm}(x, \lambda)=\psi_{\text {gen }}(x, \lambda) T_{\text {gen }}^{\mp}(\lambda) D_{\text {gen }}^{ \pm}(\lambda),
\end{array}
$$

where $S_{\text {gen }}^{ \pm}(\lambda), T_{\text {gen }}^{ \pm}(\lambda)$ and $D_{\text {gen }}^{ \pm}(\lambda)$ are the factors in the Gauss decompositions of $T_{\text {gen }}(\lambda)$ :

$$
T_{\text {gen }}(\lambda)=T_{\text {gen }}^{-}(\lambda) D_{\text {gen }}^{+}(\lambda) \hat{S}_{\text {gen }}^{+}(\lambda)=T_{\text {gen }}^{+}(\lambda) D_{\text {gen }}^{-}(\lambda) \hat{S}_{\text {gen }}^{-}(\lambda) .
$$

More specifically $S_{\text {gen }}^{+}(\lambda)$ and $T_{\text {gen }}^{+}(\lambda)$ (resp. $S_{\text {gen }}^{-}(\lambda)$ and $T_{\text {gen }}^{-}(\lambda)$ ) are upper (resp. lower) triangular matrices whose diagonal elements are equal to 1 . The diagonal matrices $D_{\text {gen }}^{+}(\lambda)$ and $D_{\text {gen }}^{-}(\lambda)$ allow analytic extension in the upper and lower half planes respectively.

The dressing factors $u_{k}(x, \lambda)$ are the simplest possible ones [47]:

$$
u_{k, \text { gen }}(x, \lambda)=\mathbb{1}+\left(c_{k}(\lambda)-1\right) P_{k}(x), \quad u_{k, \text { gen }}^{-1}(x, \lambda)=\mathbb{1}+\left(\frac{1}{c_{k}(\lambda)}-1\right) P_{k}(x),
$$

where the rank-1 projectors $P_{k}(x)$ are expressed through the regular solutions analogously to equations (3.3)-(3.5) with $\chi_{0}^{ \pm}\left(x, \lambda_{k}^{ \pm}\right)$replaced by $\chi_{0, \text { gen }}^{ \pm}\left(x, \lambda_{k}^{ \pm}\right)$

The relations between the dressed and 'naked' scattering data are the same like in equation (3.2) only now the asymptotic values $u_{\text {gen; }}(\lambda)$ are different. Assuming that all projectors $P_{k}$ have rank 1 we get:

$$
\begin{aligned}
& u_{\text {gen } ; \pm}(\lambda)=\prod_{k=1}^{N} u_{k, \text { gen } ; \pm}(\lambda), \quad u_{k, \text { gen } ; \pm}(\lambda)=\mathbb{1}+\left(c_{k}(\lambda)-1\right) P_{k, \pm}, \\
& P_{k,+}=E_{s_{k}, s_{k}}, \quad P_{k,-}=E_{p_{k}, p_{k}},
\end{aligned}
$$

where $s_{k}$ (resp. $p_{k}$ ) labels the position of the first (resp. the last) non-vanishing component of the polarization vector $\left|n_{0, k}\right\rangle$.

Using the FAS we introduce the resolvent $R_{\text {gen }}(\lambda)$ of $L_{\text {gen }}$ in the form:

$$
R_{\text {gen }}(\lambda) f(x)=\int_{-\infty}^{\infty} R_{\text {gen }}(x, y, \lambda) f(y)
$$

The kernel $R_{\text {gen }}(x, y, \lambda)$ of the resolvent is given by:

$$
R_{\text {gen }}(x, y, \lambda)= \begin{cases}R_{\text {gen }}^{+}(x, y, \lambda) & \text { for } \lambda \in \mathbb{C}^{+} \\ R_{\text {gen }}^{-}(x, y, \lambda) & \text { for } \lambda \in \mathbb{C}^{-}\end{cases}
$$

where

$$
\begin{aligned}
& R_{\text {gen }}^{ \pm}(x, y, \lambda)= \pm i \chi_{\text {gen }}^{ \pm}(x, \lambda) \Theta^{ \pm}(x-y) \hat{\chi}_{\text {gen }}^{ \pm}(y, \lambda), \\
& \Theta^{ \pm}(z)=\theta(\mp z) \Pi_{0}-\theta( \pm z)\left(\mathbb{1}-\Pi_{0}\right), \quad \Pi_{0}=\sum_{s=1}^{k} E_{s s},
\end{aligned}
$$




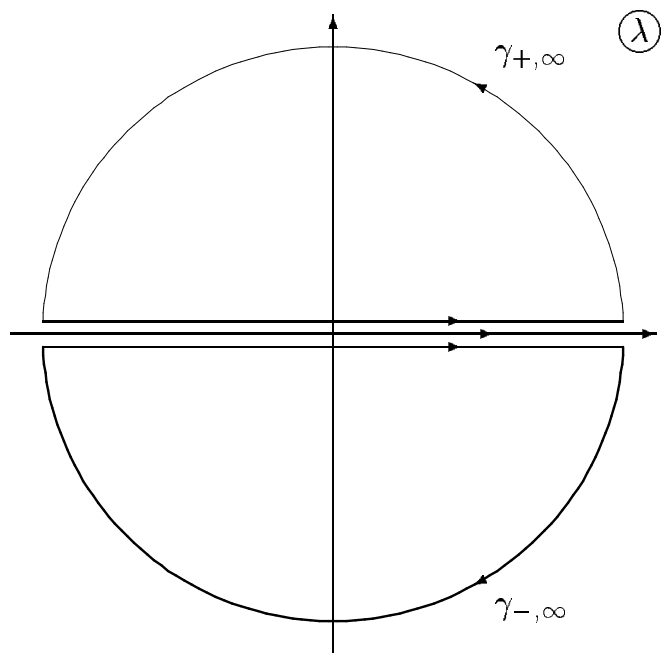

Figure 1. The contours $\gamma_{ \pm}=\mathbb{R} \cup \gamma_{ \pm \infty}$.

Theorem 1. Let $Q(x)$ be a potential of $L$ which falls off fast enough for $x \rightarrow \pm \infty$ and the corresponding RHP has a finite number of simple singularities at the points $\lambda_{j}^{ \pm} \in \mathbb{C}_{ \pm}$, i.e. $\chi_{\mathrm{gen}}^{ \pm}(x, \lambda)$ have simple poles and zeroes at $\lambda_{j}^{ \pm}$. Then

1) $R_{\text {gen }}^{ \pm}(x, y, \lambda)$ is an analytic function of $\lambda$ for $\lambda \in \mathbb{C}_{ \pm}$having pole singularities at $\lambda_{j}^{ \pm} \in \mathbb{C}_{ \pm}$;

2) $R_{\mathrm{gen}}^{ \pm}(x, y, \lambda)$ is a kernel of a bounded integral operator for $\operatorname{Im} \lambda \neq 0$;

3) $R_{\mathrm{gen}}(x, y, \lambda)$ is an uniformly bounded function for $\lambda \in \mathbb{R}$ and provides the kernel of an unbounded integral operator;

4) $R_{\mathrm{gen}}^{ \pm}(x, y, \lambda)$ satisfy the equation:

$$
L_{\text {gen }}(\lambda) R_{\text {gen }}^{ \pm}(x, y, \lambda)=\mathbb{1} \delta(x-y) .
$$

Skipping the details (see [17]) we will formulate below the completeness relation for the eigenfunctions of the Lax operator $L_{\text {gen }}$. It is derived by applying the contour integration method (see e.g. $[30,1])$ to the integral:

$$
\partial_{\text {gen }}(x, y)=\frac{1}{2 \pi i} \oint_{\gamma_{+}} d \lambda R_{\text {gen }}^{+}(x, y, \lambda)-\frac{1}{2 \pi i} \oint_{\gamma_{-}} d \lambda R_{\text {gen }}^{-}(x, y, \lambda),
$$

where the contours $\gamma_{ \pm}$are shown on the Fig. 1 and has the form:

The explicit form of the dressing factors $u_{\text {gen }}(x, \lambda)$ makes it obvious that the kernel of the resolvent has only simple poles at $\lambda=\lambda_{k}^{ \pm}$. Therefore the final form of the completeness relation for the Jost solutions of $L_{\text {gen }}$ takes the form [17]:

$$
\begin{aligned}
\delta(x- & y) \sum_{s=1}^{n} \frac{1}{a_{s}} E_{s s} \\
& =\frac{1}{2 \pi} \int_{-\infty}^{\infty} d \lambda\left\{\sum_{s=1}^{k_{0}}\left|\chi_{\text {gen }}^{[s]+}(x, \lambda)\right\rangle\left\langle\hat{\chi}_{\text {gen }}^{[s]+}(y, \lambda)\left|-\sum_{s=k_{0}+1}^{n}\right| \chi_{\text {gen }}^{[s]-}(x, \lambda)\right\rangle\left\langle\hat{\chi}_{\text {gen }}^{[s]-}(y, \lambda)\right|\right\} \\
& +\sum_{j=1}^{N}\left(\operatorname{Res}_{\lambda=\lambda_{j}^{+}} R^{+}(x, y)+\operatorname{Res}_{\lambda=\lambda_{j}^{-}} R^{-}(x, y)\right) .
\end{aligned}
$$


It is easy to check that the residues in (3.10) can be expressed by the properly normalized eigenfunctions of $L_{\text {gen }}$ corresponding to the eigenvalues $\lambda_{j}^{ \pm}[17]$.

Thus we conclude that the continuous spectrum of $L_{\text {gen }}$ has multiplicity $n$ and fills up the whole real axis $\mathbb{R}$ of the complex $\lambda$-plane; the discrete eigenvalues of $L_{\text {gen }}$ constructed using the dressing factors $u_{\text {gen }}(x, \lambda)$ are simple and the resolvent kernel $R_{\text {gen }}(x, y, \lambda)$ has poles of order one at $\lambda=\lambda_{k}^{ \pm}$.

\subsection{Resolvent and spectral decompositions for BD.I-type Lax operators}

In our case $J$ has $n$ vanishing eigenvalues which makes the problem more difficult.

We can rewrite the Lax operator in the form:

$$
\begin{aligned}
& i \frac{\partial \chi_{1}}{\partial x}+\vec{q}^{T} \vec{\chi}_{0}=\lambda \chi_{1}, \\
& i \frac{\partial \vec{\chi}_{0}}{\partial x}+\vec{q}^{*} \chi_{1}+s_{0} \vec{q} \chi_{-1}=0, \\
& i \frac{\partial \chi_{-1}}{\partial x}+\vec{q}^{\dagger} s_{0} \vec{\chi}_{0}=\lambda \chi_{-1},
\end{aligned}
$$

where we have split the eigenfunction $\chi(x, \lambda)$ of $L$ into three according to the natural blockmatrix structure compatible with $J: \chi(x, \lambda)=\left(\chi_{1}, \vec{\chi}_{0}^{T}, \chi_{-1}\right)^{T}$. Note that the equation for $\vec{\chi}_{0}$ can not be treated as eigenvalue equations; they can be formally integrated with:

$$
\vec{\chi}_{0}(x, \lambda)=\vec{\chi}_{0, \text { as }}+i \int^{x} d y\left(\vec{q}^{*} \chi_{1}+s_{0} \vec{q} \chi-1\right),
$$

which eventually casts the Lax operator into the following integro-differential system with nondegenerate $\lambda$ dependence:

$$
\begin{aligned}
& i \frac{\partial \chi_{1}}{\partial x}+i \vec{q}^{T}(x) \int^{x} d y\left(\vec{q}^{*} \chi_{1}+s_{0} \vec{q} \chi_{-1}\right)(y, \lambda)=\lambda \chi_{1}, \\
& i \frac{\partial \chi_{-1}}{\partial x}+i \vec{q}^{\dagger}(x) s_{0} \int^{x} d y\left(\vec{q}^{*} \chi_{1}+s_{0} \vec{q} \chi_{-1}\right)(y, \lambda)=-\lambda \chi_{-1} .
\end{aligned}
$$

Similarly we can treat the operator which is adjoint to $L$ whose FAS $\hat{\chi}(x, \lambda)$ are the inverse to $\chi(x, \lambda)$, i.e. $\hat{\chi}(x, \lambda)=\chi^{-1}(x, \lambda)$. Splitting each of the rows of $\hat{\chi}(x, \lambda)$ into components as follows $\hat{\chi}(x, \lambda)=\left(\hat{\chi}_{1}, \hat{\vec{\chi}}_{0}, \hat{\chi}-1\right)$ we get:

$$
\begin{aligned}
& i \frac{\partial \hat{\chi}_{1}}{\partial x}-\left(\hat{\vec{\chi}}_{0}, \vec{q}^{*}\right)-\lambda \hat{\chi}_{1}=0, \\
& i \frac{\partial \hat{\vec{\chi}}_{0}}{\partial x}-\hat{\chi}_{1} \vec{q}^{T}-\hat{\chi}_{-1} \vec{q}^{\dagger} s_{0}=0, \\
& i \frac{\partial \hat{\chi}_{-1}}{\partial x}-\left(\hat{\vec{\chi}}_{0}, s_{0} \vec{q}\right)-\lambda \hat{\chi}-1=0 .
\end{aligned}
$$

Again the equation for $\hat{\vec{\chi}}_{0}$ can be formally integrated with:

$$
\hat{\vec{\chi}}_{0}(x, \lambda)=\hat{\vec{\chi}}_{0, \text { as }}+i \int^{x} d y\left(\hat{\chi}_{1}(y, \lambda) \vec{q}^{T}(y)+\hat{\chi}_{-1}(y, \lambda) \vec{q}^{\dagger}(y) s_{0}\right) .
$$

Now we get the following integro-differential system with non-degenerate $\lambda$ dependence

$$
i \frac{\partial \hat{\chi}_{1}}{\partial x}-i \int^{x} d y\left(\hat{\chi}_{1}(y, \lambda)\left(\vec{q}^{T}(y), \vec{q}^{*}(x)\right)+\hat{\chi}_{-1}(y, \lambda)\left(\vec{q}^{\dagger}(y) s_{0} \vec{q}^{*}(x)\right)\right)+\lambda \hat{\chi}_{1}=0,
$$




$$
i \frac{\partial \hat{\chi}_{-1}}{\partial x}-i \int^{x} d y\left(\hat{\chi}_{1}(y, \lambda)\left(\vec{q}^{T}(y) s_{0} \vec{q}(x)\right)+\hat{\chi}_{-1}(y, \lambda)\left(\vec{q}^{\dagger}(y), \vec{q}(x)\right)\right)-\lambda \hat{\chi}_{-1}=0 .
$$

Now we are ready to generalize the standard approach to the case of BD.I-type Lax operators.

The kernel $R(x, y, \lambda)$ of the resolvent is given by:

$$
R(x, y, \lambda)= \begin{cases}R^{+}(x, y, \lambda) & \text { for } \lambda \in \mathbb{C}^{+} \\ R^{-}(x, y, \lambda) & \text { for } \lambda \in \mathbb{C}^{-}\end{cases}
$$

where

$$
\begin{aligned}
& R^{ \pm}(x, y, \lambda)= \pm i \chi^{ \pm}(x, \lambda) \Theta^{ \pm}(x-y) \hat{\chi}^{ \pm}(y, \lambda), \\
& \Theta^{ \pm}(z)=\theta(\mp z) E_{11}-\theta( \pm z)\left(\mathbb{1}-E_{11}\right) .
\end{aligned}
$$

The completeness relation for the eigenfunctions of the Lax operator $L$ is derived by applying the contour integration method (see e.g. $[30,1]$ ) to the integral:

$$
g^{\prime}(x, y)=\frac{1}{2 \pi i} \oint_{\gamma_{+}} d \lambda \Pi_{1} R^{+}(x, y, \lambda)-\frac{1}{2 \pi i} \oint_{\gamma_{-}} d \lambda \Pi_{1} R^{-}(x, y, \lambda),
$$

where the contours $\gamma_{ \pm}$are shown on the Fig. 1 and $\Pi_{1}=E_{11}+E_{n+2, n+2}$. Using equations (3.8) and (3.9) we are able to check that the kernel of the resolvent has poles of second order at $\lambda=\lambda_{k}^{ \pm}$. Therefore the completeness relation takes the form:

$$
\begin{aligned}
\Pi_{1} \delta(x-y)= & \frac{1}{2 \pi} \int_{-\infty}^{\infty} d \lambda \Pi_{1}\left\{\left|\chi_{\text {gen }}^{[1]+}(x, \lambda)\right\rangle\left\langle\hat{\chi}_{\text {gen }}^{[1]+}(y, \lambda)|-| \chi_{\text {gen }}^{[n+2]-}(x, \lambda)\right\rangle\left\langle\hat{\chi}_{\text {gen }}^{[n+2]-}(y, \lambda)\right|\right\} \\
& +2 i \sum_{j=1}^{N}\left\{\operatorname{Res}_{\lambda=\lambda_{k}^{+}} R^{+}(x, y, \lambda)+\operatorname{Res}_{\lambda=\lambda_{k}^{-}} R^{-}(x, y, \lambda)\right\}
\end{aligned}
$$

where

$$
\operatorname{Res}_{\lambda=\lambda_{k}^{ \pm}} R^{ \pm}(x, y, \lambda)= \pm\left(\lambda_{k}^{-}-\lambda_{k}^{+}\right) \Pi_{1}\left(\chi^{+,(k)}(x) \hat{\dot{\chi}}^{+,(k)}(y)+\dot{\chi}^{+,(k)}(x) \hat{\chi}^{+,(k)}(y)\right) .
$$

In other words the continuous spectrum of $L$ has multiplicity 2 and fills up the whole real axis $\mathbb{R}$ of the complex $\lambda$-plane; the discrete eigenvalues of $L$ constructed using the dressing factors $u(x, \lambda)(3.7)$ lead to second order poles of resolvent kernel $R_{\text {gen }}(x, y, \lambda)$ at $\lambda=\lambda_{k}^{ \pm}$.

\section{Resolvent and spectral decompositions in the adjoint representation of $\mathfrak{g} \simeq B_{r}$}

The simplest realization of $L$ in the adjoint representation is to make use of the adjoint action of $Q(x)-\lambda J$ on $\mathfrak{g}:$

$$
L_{\mathrm{ad}} e_{\mathrm{ad}} \equiv i \frac{\partial e_{\mathrm{ad}}}{\partial x}+\left[Q(x)-\lambda J_{\mathrm{ad}}, e_{\mathrm{ad}}(x, \lambda)\right]=0 .
$$

Note that the eigenfunctions of $L_{\text {ad }}$ take values in the Lie algebra $\mathfrak{g}$. They are known also as the 'squared solutions' of $L$ and appear in a natural way in the analysis of the transform from the potential $Q(x, t)$ to the scattering data of $L,[1]$; see also [14, 17] and the numerous references therein.

The idea for the interpretation of the ISM as a generalized Fourier transform was launched in [1]. It is based on the Wronskian relations which allow one to maps the potential $Q(x, t)$ onto 
the minimal sets of scattering data $\mathcal{T}_{i}$. These ideas have been generalized also to the symmetric spaces, see [14, 25, 22] and the references therein.

The 'squared solutions' that play the role of generalized exponentials are determined by the FAS and the Cartan-Weyl basis of the corresponding algebra as follows. First we introduce:

$$
e_{\alpha, \text { ad }}^{ \pm}(x, \lambda)=\chi^{ \pm} E_{\alpha} \hat{\chi}^{ \pm}(x, \lambda), \quad e_{j, \text { ad }}^{ \pm}(x, \lambda)=\chi^{ \pm} H_{j} \hat{\chi}^{ \pm}(x, \lambda),
$$

where $\chi^{ \pm}(x, \lambda)$ are the FAS of $L$ and $E_{\alpha}, H_{j}$ form the Cartan-Weyl basis of $\mathfrak{g}$. Next we note that in the adjoint representation $J_{\mathrm{ad}} \cdot \equiv \operatorname{ad} J \cdot \equiv[J, \cdot]$ has kernel. Just like in the previous section we have to project out that kernel, i.e. we need to introduce the projector:

$$
\pi_{J} X \equiv \operatorname{ad}_{J}^{-1} \operatorname{ad}_{J} X
$$

for any $X \in \mathfrak{g}$. In particular, choosing $\mathfrak{g} \simeq s o(2 r+1)$ and $J$ as in equation (2.1) we find that the potential $Q$ provides a generic element of the image of $\pi_{J}$, i.e. $\pi_{J} Q \equiv Q$.

Next, the analysis of the Wronskian relations allows one to introduce two sets of squared solutions:

$$
\boldsymbol{\Psi}_{\alpha}^{ \pm}=\pi_{J}\left(\chi^{ \pm}(x, \lambda) E_{\alpha} \hat{\chi}^{ \pm}(x, \lambda)\right), \quad \boldsymbol{\Phi}_{\alpha}^{ \pm}=\pi_{J}\left(\chi^{ \pm}(x, \lambda) E_{-\alpha} \hat{\chi}^{ \pm}(x, \lambda)\right), \quad \alpha \in \Delta_{1}^{+} .
$$

We remind that the set $\Delta_{1}$ contains all roots of $\operatorname{so}(r+1)$ for which $\alpha(J) \neq 0$.

Let us introduce the sets of 'squared solutions':

$$
\begin{aligned}
& \{\boldsymbol{\Psi}\}=\{\boldsymbol{\Psi}\}_{\mathrm{c}} \cup\{\boldsymbol{\Psi}\}_{\mathrm{d}}, \quad\{\boldsymbol{\Phi}\}=\{\boldsymbol{\Phi}\}_{\mathrm{c}} \cup\{\boldsymbol{\Phi}\}_{\mathrm{d}}, \\
& \{\boldsymbol{\Psi}\}_{\mathrm{c}} \equiv\left\{\boldsymbol{\Psi}_{\alpha}^{+}(x, \lambda), \boldsymbol{\Psi}_{-\alpha}^{-}(x, \lambda), i<r, \lambda \in \mathbb{R}\right\}, \\
& \{\boldsymbol{\Psi}\}_{\mathrm{d}} \equiv\left\{\boldsymbol{\Psi}_{\alpha ; j}^{+}(x), \dot{\boldsymbol{\Psi}}_{\alpha ; j}^{+}(x), \boldsymbol{\Psi}_{-\alpha ; j}^{-}(x), \dot{\boldsymbol{\Psi}}_{-\alpha ; j}^{-}(x)\right\}_{j=1}^{N}, \\
& \{\boldsymbol{\Phi}\}_{\mathrm{c}} \equiv\left\{\boldsymbol{\Phi}_{-\alpha}^{+}(x, \lambda), \boldsymbol{\Phi}_{\alpha}^{-}(x, \lambda), i<r, \lambda \in \mathbb{R}\right\}, \\
& \{\boldsymbol{\Phi}\}_{\mathrm{d}} \equiv\left\{\boldsymbol{\Phi}_{-\alpha ; j}^{+}(x), \dot{\boldsymbol{\Phi}}_{-\alpha ; j}^{+}(x), \boldsymbol{\Phi}_{\alpha ; j}^{-}(x), \dot{\boldsymbol{\Phi}}_{\alpha ; j}^{-}(x)\right\}_{j=1}^{N},
\end{aligned}
$$

where the subscripts ' $c$ ' and 'd' refer to the continuous and discrete spectrum of $L$.

Each of the above two sets are complete sets of functions in the space of allowed potentials. This fact can be proved by applying the contour integration method to the integral

$$
\partial_{G}(x, y)=\frac{1}{2 \pi i} \oint_{\gamma_{+}} d \lambda G^{+}(x, y, \lambda)-\frac{1}{2 \pi i} \oint_{\gamma_{-}} d \lambda G^{-}(x, y, \lambda),
$$

where the Green function is defined by:

$$
\begin{aligned}
& G^{ \pm}(x, y, \lambda)=G_{1}^{ \pm}(x, y, \lambda) \theta(y-x)-G_{2}^{ \pm}(x, y, \lambda) \theta(x-y), \\
& G_{1}^{ \pm}(x, y, \lambda)=\sum_{\alpha \in \Delta_{1}^{+}} \boldsymbol{\Psi}_{ \pm \alpha}^{ \pm}(x, \lambda) \otimes \mathbf{\Phi}_{\mp \alpha}^{ \pm}(y, \lambda), \\
& G_{2}^{ \pm}(x, y, \lambda)=\sum_{\alpha \in \Delta_{0} \cup \Delta_{1}^{-}} \boldsymbol{\Phi}_{ \pm \alpha}^{ \pm}(x, \lambda) \otimes \boldsymbol{\Psi}_{\mp \alpha}^{ \pm}(y, \lambda)+\sum_{j=1}^{r} \boldsymbol{h}_{j}^{ \pm}(x, \lambda) \otimes \boldsymbol{h}_{j}^{ \pm}(y, \lambda), \\
& \boldsymbol{h}_{j}^{ \pm}(x, \lambda)=\chi^{ \pm}(x, \lambda) H_{j} \hat{\chi}^{ \pm}(x, \lambda) .
\end{aligned}
$$

Skipping the details we give the result $[25,27]$ :

$$
\delta(x-y) \Pi_{0 J}=\frac{1}{\pi} \int_{-\infty}^{\infty} d \lambda\left(G_{1}^{+}(x, y, \lambda)-G_{1}^{-}(x, y, \lambda)\right)
$$




$$
-2 i \sum_{j=1}^{N}\left(G_{1, j}^{+}(x, y)+G_{1, j}^{-}(x, y)\right),
$$

where

$$
\begin{aligned}
& \Pi_{0 J}=\sum_{\alpha \in \Delta_{1}^{+}}\left(E_{\alpha} \otimes E_{-\alpha}-E_{-\alpha} \otimes E_{\alpha}\right), \\
& G_{1, j^{ \pm}}(x, y)=\sum_{\alpha \in \Delta_{1}^{+}}\left(\dot{\Psi}_{ \pm \alpha ; j}^{ \pm}(x) \otimes \boldsymbol{\Phi}_{\mp \alpha ; j}^{ \pm}(y)+\boldsymbol{\Psi}_{ \pm \alpha ; j}^{ \pm}(x) \otimes \dot{\boldsymbol{\Phi}}_{\mp \alpha ; j}^{ \pm}(y)\right) .
\end{aligned}
$$

\subsection{Expansion over the 'squared solutions'}

The completeness relation of the 'squared solutions' allows one to expand any function over the 'squared solutions'

Using the Wronskian relations one can derive the expansions over the 'squared solutions' of two important functions. Skipping the calculational details we formulate the results [25]. The expansion of $Q(x)$ over the systems $\left\{\boldsymbol{\Phi}^{ \pm}\right\}$and $\left\{\boldsymbol{\Psi}^{ \pm}\right\}$takes the form:

$$
\begin{aligned}
Q(x)= & \frac{i}{\pi} \int_{-\infty}^{\infty} \mathrm{d} \lambda \sum_{\alpha \in \Delta_{1}^{+}}\left(\tau_{\alpha}^{+}(\lambda) \boldsymbol{\Phi}_{\alpha}^{+}(x, \lambda)-\tau_{\alpha}^{-}(\lambda) \boldsymbol{\Phi}_{-\alpha}^{-}(x, \lambda)\right) \\
& +2 \sum_{k=1}^{N} \sum_{\alpha \in \Delta_{1}^{+}}\left(\tau_{\alpha ; j}^{+} \boldsymbol{\Phi}_{\alpha ; j}^{+}(x)+\tau_{\alpha ; j}^{-} \boldsymbol{\Phi}_{-\alpha ; j}^{-}(x)\right), \\
Q(x)= & -\frac{\mathrm{i}}{\pi} \int_{-\infty}^{\infty} \mathrm{d} \lambda \sum_{\alpha \in \Delta_{1}^{+}}\left(\rho_{\alpha}^{+}(\lambda) \boldsymbol{\Psi}_{-\alpha}^{+}(x, \lambda)-\rho_{\alpha}^{-}(\lambda) \boldsymbol{\Psi}_{\alpha}^{-}(x, \lambda)\right) \\
& -2 \sum_{k=1}^{N} \sum_{\alpha \in \Delta_{1}^{+}}\left(\rho_{\alpha ; j}^{+} \boldsymbol{\Psi}_{-\alpha ; j}^{+}(x)+\rho_{\alpha ; j}^{-} \mathbf{\Psi}_{\alpha ; j}^{-}(x)\right) .
\end{aligned}
$$

The next expansion is of $\operatorname{ad}_{J}^{-1} \delta Q(x)$ over the systems $\left\{\boldsymbol{\Phi}^{ \pm}\right\}$and $\left\{\boldsymbol{\Psi}^{ \pm}\right\}$:

$$
\begin{aligned}
\operatorname{ad}_{J}^{-1} \delta Q(x)= & \frac{\mathrm{i}}{2 \pi} \int_{-\infty}^{\infty} \mathrm{d} \lambda \sum_{\alpha \in \Delta_{1}^{+}}\left(\delta \tau_{\alpha}^{+}(\lambda) \boldsymbol{\Phi}_{\alpha}^{+}(x, \lambda)+\delta \tau_{\alpha}^{-}(\lambda) \boldsymbol{\Phi}_{-\alpha}^{-}(x, \lambda)\right) \\
& +\sum_{k=1}^{N} \sum_{\alpha \in \Delta_{1}^{+}}\left(\delta W_{\alpha ; j}^{+}(x)-\delta^{\prime} W_{-\alpha ; j}^{-}(x)\right), \\
\operatorname{ad}_{J}^{-1} \delta Q(x)= & \frac{\mathrm{i}}{2 \pi} \int_{-\infty}^{\infty} \mathrm{d} \lambda \sum_{\alpha \in \Delta_{1}^{+}}\left(\delta \rho_{\alpha}^{+}(\lambda) \boldsymbol{\Psi}_{-\alpha}^{+}(x, \lambda)+\delta \rho_{\alpha}^{-}(\lambda) \Psi_{\alpha}^{-}(x, \lambda)\right) \\
& +\sum_{k=1}^{N} \sum_{\alpha \in \Delta_{1}^{+}}\left(\delta \tilde{W}_{-\alpha ; j}^{+}(x)-\delta \tilde{W}_{\alpha ; j}^{-}(x)\right),
\end{aligned}
$$

where

$$
\begin{aligned}
& \delta W_{ \pm \alpha ; j}^{ \pm}(x)=\delta \lambda_{j}^{ \pm} \tau_{\alpha ; j}^{ \pm} \dot{\boldsymbol{\Phi}}_{ \pm \alpha ; j}^{ \pm}(x)+\delta \tau_{\alpha ; j}^{ \pm} \boldsymbol{\Phi}_{ \pm \alpha ; j}^{ \pm}(x), \\
& \delta \tilde{W}_{\mp \alpha ; j}^{ \pm}(x)=\delta \lambda_{j}^{ \pm} \rho_{\alpha ; j}^{ \pm} \dot{\boldsymbol{\Psi}}_{\mp \alpha ; j}^{ \pm}(x)+\delta \rho_{\alpha ; j}^{ \pm} \boldsymbol{\Psi}_{\mp \alpha ; j}^{ \pm}(x)
\end{aligned}
$$

and $\boldsymbol{\Phi}_{ \pm \alpha ; j}^{ \pm}(x)=\boldsymbol{\Phi}_{ \pm \alpha}^{ \pm}\left(x, \lambda_{j}^{ \pm}\right), \dot{\boldsymbol{\Phi}}_{ \pm \alpha ; j}^{ \pm}(x)=\left.\partial_{\lambda} \boldsymbol{\Phi}_{ \pm \alpha}^{ \pm}(x, \lambda)\right|_{\lambda=\lambda_{j}^{ \pm}}$. 
The expansions (4.2), (4.3) is another way to establish the one-to-one correspondence between $Q(x)$ and each of the minimal sets of scattering data $\mathcal{T}_{1}$ and $\mathcal{T}_{2}$ (2.10). Likewise the expansions (4.4), (4.5) establish the one-to-one correspondence between the variation of the potential $\delta Q(x)$ and the variations of the scattering data $\delta \mathcal{T}_{1}$ and $\delta \mathcal{T}_{2}$.

The expansions (4.4), (4.5) have a special particular case when one considers the class of variations of $Q(x, t)$ due to the evolution in $t$. Then

$$
\delta Q(x, t) \equiv Q(x, t+\delta t)-Q(x, t)=\frac{\partial Q}{\partial t} \delta t+\mathcal{O}\left((\delta t)^{2}\right) .
$$

Assuming that $\delta t$ is small and keeping only the first order terms in $\delta t$ we get the expansions for $\operatorname{ad}_{J}^{-1} Q_{t}$. They are obtained from (4.4), (4.5) by replacing $\delta \rho_{\alpha}^{ \pm}(\lambda)$ and $\delta \tau_{\alpha}^{ \pm}(\lambda)$ by $\partial_{t} \rho_{\alpha}^{ \pm}(\lambda)$ and $\partial_{t} \rho_{\alpha}^{ \pm}(\lambda)$.

\subsection{The generating operators}

To complete the analogy between the standard Fourier transform and the expansions over the 'squared solutions' we need the analogs of the operator $D_{0}=-\mathrm{id} / \mathrm{d} x$. The operator $D_{0}$ is the one for which $\mathrm{e}^{\mathrm{i} \lambda x}$ is an eigenfunction: $D_{0} \mathrm{e}^{\mathrm{i} \lambda x}=\lambda \mathrm{e}^{\mathrm{i} \lambda x}$. Therefore it is natural to introduce the generating operators $\Lambda_{ \pm}$through:

$$
\begin{array}{lll}
\left(\Lambda_{+}-\lambda\right) \boldsymbol{\Psi}_{-\alpha}^{+}(x, \lambda)=0, & \left(\Lambda_{+}-\lambda\right) \boldsymbol{\Psi}_{\alpha}^{-}(x, \lambda)=0, & \left(\Lambda_{+}-\lambda_{j}^{ \pm}\right) \boldsymbol{\Psi}_{\mp \alpha ; j}^{+}(x)=0, \\
\left(\Lambda_{-}-\lambda\right) \boldsymbol{\Phi}_{\alpha}^{+}(x, \lambda)=0, & \left(\Lambda_{-}-\lambda\right) \boldsymbol{\Phi}_{-\alpha}^{-}(x, \lambda)=0, & \left(\Lambda_{+}-\lambda_{j}^{ \pm}\right) \boldsymbol{\Phi}_{ \pm \alpha ; j}^{+}(x)=0,
\end{array}
$$

where the generating operators $\Lambda_{ \pm}$are given by:

$$
\Lambda_{ \pm} X(x) \equiv \operatorname{ad}_{J}^{-1}\left(\mathrm{i} \frac{\mathrm{d} X}{\mathrm{~d} x}+\mathrm{i}\left[Q(x), \int_{ \pm \infty}^{x} \mathrm{~d} y[Q(y), X(y)]\right]\right) .
$$

The rest of the squared solutions are not eigenfunctions of neither $\Lambda_{+}$nor $\Lambda_{-}$:

$$
\begin{array}{ll}
\left(\Lambda_{+}-\lambda_{j}^{+}\right) \dot{\boldsymbol{\Psi}}_{-\alpha ; j}^{+}(x)=\boldsymbol{\Psi}_{-\alpha ; j}^{+}(x), & \left(\Lambda_{+}-\lambda_{j}^{-}\right) \dot{\boldsymbol{\Psi}}_{\alpha ; j}^{-}(x)=\boldsymbol{\Psi}_{\alpha ; j}^{-}(x), \\
\left(\Lambda_{-}-\lambda_{j}^{+}\right) \dot{\boldsymbol{\Phi}}_{i r ; j}^{+}(x)=\boldsymbol{\Phi}_{\alpha ; j}^{+}(x), & \left(\Lambda_{-}-\lambda_{j}^{-}\right) \dot{\boldsymbol{\Phi}}_{\alpha ; j}^{-}(x)=\boldsymbol{\Phi}_{\alpha ; j}^{-}(x),
\end{array}
$$

i.e., $\dot{\mathbf{\Psi}}_{\alpha ; j}^{+}(x)$ and $\dot{\boldsymbol{\Phi}}_{\alpha ; j}^{+}(x)$ are adjoint eigenfunctions of $\Lambda_{+}$and $\Lambda_{-}$. This means that $\lambda_{j}^{ \pm}, j=$ $1, \ldots, N$ are also the discrete eigenvalues of $\Lambda_{ \pm}$but the corresponding eigenspaces of $\Lambda_{ \pm}$have double the dimensions of the ones of $L$; now they are spanned by both $\boldsymbol{\Psi}_{\mp \alpha ; j}^{ \pm}(x)$ and $\dot{\boldsymbol{\Psi}}_{\mp \alpha ; j}^{ \pm}(x)$. Thus the sets $\{\Psi\}$ and $\{\Phi\}$ are the complete sets of eigen- and adjoint functions of $\Lambda_{+}$and $\Lambda_{-}$.

Therefore the completeness relation (4.1) can be viewed as the spectral decompositions of the recursion operators $\Lambda_{ \pm}$. It is also obvious that the continuous spectrum of these operators fills up the real axis $\mathbb{R}$ and has multiplicity $2 n$; the discrete spectrum consists of the eigenvalues $\lambda_{k}^{ \pm}$ and each of them has multiplicity 2.

\section{Resolvent and spectral decompositions in the spinor representation of $\mathfrak{g} \simeq B_{r}$}

Using the general theory one can calculate the explicit form of the Cartan-Weyl basis in the spinor representations. In Appendices B and C below we give the results for $r=2$ and $r=3$. Therefore in the spinor representation the Lax operators take the form:

$$
L_{\mathrm{sp}} \psi_{\mathrm{sp}}=i \frac{\partial \psi_{\mathrm{sp}}}{\partial x}+\left(Q_{\mathrm{sp}}-\lambda J_{\mathrm{sp}}\right) \psi_{\mathrm{sp}}(x, \lambda)=0
$$


where $Q_{\mathrm{sp}}(x, t)$ and $J_{\mathrm{sp}}$ are $2^{r} \times 2^{r}$ matrices of the form:

$$
Q_{\mathrm{sp}}=\left(\begin{array}{cc}
0 & \boldsymbol{q} \\
\boldsymbol{q}^{\dagger} & 0
\end{array}\right), \quad J_{\mathrm{sp}}=\frac{1}{2}\left(\begin{array}{cc}
\mathbb{1}_{2} & 0 \\
0 & -\mathbb{1}_{2}
\end{array}\right),
$$

where the explicit form of $\boldsymbol{q}(x)$ for $r=2$ and $r=3$ is given by equations (B.1) and (C.1) below.

It is well known [5] that the spinor representations of $s o(2 r+1)$ are realized by symplectic (resp. orthogonal) matrices if $r(r+1) / 2$ is odd (resp. even). Thus in what follows we will view the spinor representations of $s o(2 r+1)$ as typical representations of $s p\left(2^{r}\right)$ (resp. $s o\left(2^{r}\right)$ ) algebra. Combined with the corresponding value of $J_{\mathrm{sp}}$ (B.1) one can conclude that that the Lax operator $L_{\mathrm{sp}}$ for odd values of $r(r+1) / 2$ can be related to the C.III-type symmetric spaces. The potential $Q_{\mathrm{sp}}(x)$ however is not a generic one; it may be obtained from the generic potential as a special reduction, which picks up $s o(2 r+1)$ as the subalgebra of $s p\left(2^{r}\right)$. Below we will construct an automorphism whose kernel will pick up $s o(2 r+1)$ as a subalgebra of $s p\left(2^{r}\right)$.

Similarly the Lax operator $L_{\mathrm{sp}}$ above for even values of $r(r+1) / 2$ can be related to the $s o\left(2^{r}\right)$ algebra. The element $J_{\mathrm{sp}}$ (B.1) is characteristic for the D.III-type symmetric spaces. The potential $Q_{\mathrm{sp}}(x)$ may be obtained from the generic potential as a special reduction, which picks up $s o(2 r+1)$ as the subalgebra of $s o\left(2^{r}\right)$.

The spectral problem (5.1) is technically more simple to treat. It has the form of block-matrix AKNS which means that the corresponding Jost solutions and FAS are determined as follows:

$$
\begin{aligned}
& \psi(x, \lambda) \underset{x \rightarrow \infty}{\simeq} e^{-i \lambda J x}, \quad \phi(x, \lambda) \underset{x \rightarrow-\infty}{\simeq} e^{-i \lambda J x}, \quad T(\lambda)=\left(\begin{array}{c}
\boldsymbol{a}^{+}-\boldsymbol{b}^{-} \\
\boldsymbol{b}^{+} \boldsymbol{a}^{-}
\end{array}\right), \\
& \psi(x, \lambda)=\left(\psi^{-}(x, \lambda), \psi^{+}(x, \lambda)\right), \quad \phi(x, \lambda)=\left(\phi^{+}(x, \lambda), \phi^{-}(x, \lambda)\right), \\
& \chi^{+}(x, \lambda)=\left(\phi^{+}(x, \lambda), \psi^{+}(x, \lambda)\right), \quad \chi^{-}(x, \lambda)=\left(\psi^{-}(x, \lambda), \phi^{-}(x, \lambda)\right) .
\end{aligned}
$$

\subsection{The Gauss factors in the spinor representation}

The spectral theory of the Lax operators related to the symmetric spaces of C.III and D.III types were developed in $[20,28]$. What is different here is the special choice of the rank and the additional reduction $\pi_{\mathrm{B}_{\mathrm{r}}}$ which picks up the spinor representation of $s o(2 r+1)$. In our considerations below we will assume that this reduction is applied. So though all our $2^{r} \times 2^{r}$ matrices are split into blocks of dimension $2^{r-1} \times 2^{r-1}$, the corresponding group (resp. algebraic) elements belong to the (spinor representation of) group $S O(2 r+1)$ (resp. algebra $s o(2 r+1)$ ). Thus we define the FAS of $L_{\mathrm{sp}}$ by:

$$
\begin{aligned}
& \chi_{\mathrm{sp}}^{+}(x, \lambda) \equiv\left(\left|\phi^{+}\right\rangle,\left|\psi^{+} \hat{c}^{+}\right\rangle\right)(x, \lambda)=\phi(x, \lambda) \boldsymbol{S}_{\mathrm{sp}}^{+}(\lambda)=\psi_{\mathrm{sp}}(x, \lambda) \boldsymbol{T}_{\mathrm{sp}}^{-}(\lambda) D_{\mathrm{sp}}^{+}(\lambda), \\
& \chi_{\mathrm{sp}}^{-}(x, \lambda) \equiv\left(\left|\psi^{-} \hat{c}^{-}\right\rangle,\left|\phi^{-}\right\rangle\right)(x, \lambda)=\phi(x, \lambda) \boldsymbol{S}_{\mathrm{sp}}^{-}(\lambda)=\psi_{\mathrm{sp}}(x, \lambda) \boldsymbol{T}_{\mathrm{sp}}^{+}(\lambda) D_{\mathrm{sp}}^{-}(\lambda),
\end{aligned}
$$

where the block-triangular functions $\boldsymbol{S}_{\mathrm{sp}}^{ \pm}(\lambda)$ and $\boldsymbol{T}_{\mathrm{sp}}^{ \pm}(\lambda)$ are given by:

$$
\begin{array}{ll}
\boldsymbol{S}_{\mathrm{sp}}^{+}(\lambda)=\left(\begin{array}{cc}
\mathbb{1} \boldsymbol{d}^{-} \hat{\boldsymbol{c}}^{+}(\lambda) \\
0 & \mathbb{1}
\end{array}\right), & \boldsymbol{T}_{\mathrm{sp}}^{-}(\lambda)=\left(\begin{array}{cc}
\mathbb{1} & 0 \\
\boldsymbol{b}^{+} \hat{\boldsymbol{a}}^{+}(\lambda) & \mathbb{1}
\end{array}\right), \\
\boldsymbol{S}_{\mathrm{sp}}^{-}(\lambda)=\left(\begin{array}{cc}
\mathbb{1} & 0 \\
-\boldsymbol{d}^{+} \hat{\boldsymbol{c}}^{-}(\lambda) & \mathbb{1}
\end{array}\right), & \boldsymbol{T}_{\mathrm{sp}}^{+}(\lambda)=\left(\begin{array}{cc}
\mathbb{1}-\boldsymbol{b}^{-} \hat{\boldsymbol{a}}^{-}(\lambda) \\
0 & \mathbb{1}
\end{array}\right) .
\end{array}
$$

The matrices $D_{\mathrm{sp}}^{ \pm}(\lambda)$ are block-diagonal and equal:

$$
D_{\mathrm{sp}}^{+}(\lambda)=\left(\begin{array}{cc}
\boldsymbol{a}^{+}(\lambda) & 0 \\
0 & \hat{\boldsymbol{c}}^{+}(\lambda)
\end{array}\right), \quad D_{\mathrm{sp}}^{-}(\lambda)=\left(\begin{array}{cc}
\hat{\boldsymbol{c}}^{-}(\lambda) & 0 \\
0 & \boldsymbol{a}^{-}(\lambda)
\end{array}\right) .
$$

The supper scripts \pm here refer to their analyticity properties for $\lambda \in \mathbb{C}_{ \pm}$. 
All factors $\boldsymbol{S}_{\mathrm{sp}}^{ \pm}, \boldsymbol{T}_{\mathrm{sp}}^{ \pm}$and $D_{\mathrm{sp}}^{ \pm}$take values in the spinor representation of the group $S O(2 r+1)$ and are determined by the minimal sets of scattering data (2.15). Besides, since

$$
\begin{aligned}
& T_{\mathrm{sp}}(\lambda)=\boldsymbol{T}_{\mathrm{sp}}^{-}(\lambda) D_{\mathrm{sp}}^{+}(\lambda) \hat{\boldsymbol{S}}_{\mathrm{sp}}^{+}(\lambda)=\boldsymbol{T}_{\mathrm{sp}}^{+}(\lambda) D_{\mathrm{sp}}^{-}(\lambda) \hat{\boldsymbol{S}}_{\mathrm{sp}}^{-}(\lambda), \\
& \hat{T}_{\mathrm{sp}}(\lambda)=\boldsymbol{S}_{\mathrm{sp}}^{+}(\lambda) \hat{D}_{\mathrm{sp}}^{+}(\lambda) \hat{\boldsymbol{T}}_{\mathrm{sp}}^{-}(\lambda)=\boldsymbol{S}_{\mathrm{sp}}^{-}(\lambda) \hat{D}_{\mathrm{sp}}^{-}(\lambda) \hat{\boldsymbol{T}}_{\mathrm{sp}}^{+}(\lambda),
\end{aligned}
$$

we can view the factors $\boldsymbol{S}_{\mathrm{sp}}^{ \pm}, \boldsymbol{T}_{\mathrm{sp}}^{ \pm}$and $D_{\mathrm{sp}}^{ \pm}$as generalized Gauss decompositions (see [33]) of $T_{\mathrm{sp}}(\lambda)$ and its inverse.

From equations (5.2), (5.3) one can derive:

$$
\begin{array}{ll}
\chi_{\mathrm{sp}}^{+}(x, \lambda)=\chi_{\mathrm{sp}}^{-}(x, \lambda) G_{0, \mathrm{sp}}(\lambda), & \chi_{\mathrm{sp}}^{-}(x, \lambda)=\chi_{\mathrm{sp}}^{+}(x, \lambda) \hat{G}_{0, \mathrm{sp}}(\lambda), \\
G_{0, \mathrm{sp}}(\lambda)=\left(\begin{array}{cc}
\mathbb{1} & \tau^{+} \\
\tau^{-} & \mathbb{1}+\tau^{-} \tau^{+}
\end{array}\right), & \hat{G}_{0, \mathrm{sp}}(\lambda)=\left(\begin{array}{cc}
\mathbb{1}+\tau^{+} \tau^{-} & -\tau^{+} \\
-\tau^{-} & \mathbb{1}
\end{array}\right)
\end{array}
$$

valid for $\lambda \in \mathbb{R}$. Below we introduce:

$$
X_{\mathrm{sp}}^{ \pm}(x, \lambda)=\chi_{\mathrm{sp}}^{ \pm}(x, \lambda) \mathrm{e}^{i \lambda J x} .
$$

Strictly speaking it is $X_{\mathrm{sp}}^{ \pm}(x, \lambda)$ that allow analytic extension for $\lambda \in \mathbb{C}_{ \pm}$. They have also another nice property, namely their asymptotic behavior for $\lambda \rightarrow \pm \infty$ is given by:

$$
\lim _{\lambda \rightarrow \infty} X_{\mathrm{sp}}^{ \pm}(x, \lambda)=\mathbb{1}
$$

Along with $X_{\mathrm{sp}}^{ \pm}(x, \lambda)$ we can use another set of FAS $\tilde{X}_{\mathrm{sp}}^{ \pm}(x, \lambda)=X_{\mathrm{sp}}^{ \pm}(x, \lambda) \hat{D}_{\mathrm{sp}}^{ \pm}$, which also satisfy equation (5.7) due to the fact that:

$$
\lim _{\lambda \rightarrow \infty} D_{\mathrm{sp}}^{ \pm}(\lambda)=\mathbb{1}
$$

The equations (5.4) and (5.5) can be written down as:

$$
X_{\mathrm{sp}}^{+}(x, \lambda)=X_{\mathrm{sp}}^{-}(x, \lambda) G_{\mathrm{sp}}(x, \lambda), \quad \lambda \in \mathbb{R}
$$

with

$$
\tilde{G}_{\mathrm{sp}}(x, \lambda)=\mathrm{e}^{-i \lambda J x} \tilde{G}_{0, \mathrm{sp}}(\lambda) e^{i \lambda J x}, \quad \tilde{G}_{0, \mathrm{sp}}(\lambda)=\left(\begin{array}{cc}
\mathbb{1}+\rho^{-} \rho^{+} & \rho^{-} \\
\rho^{+} & \mathbb{1}
\end{array}\right) .
$$

Equations (5.8) combined with (5.7) are known as a Riemann-Hilbert problem (RHP) with canonical normalization [13]. It has unique regular solution; the matrix-valued solutions $X_{0, \mathrm{sp}}^{+}(x, \lambda)$ and $X_{0, \mathrm{sp}}^{-}(x, \lambda)$ of $(5.8),(5.7)$ is called regular if $\operatorname{det} X_{0, \mathrm{sp}}^{ \pm}(x, \lambda)$ does not vanish for any $\lambda \in \mathbb{C}_{ \pm}$.

One can derive the following integral decomposition for $X_{\mathrm{sp}}^{ \pm}(x, \lambda)$ :

$$
\begin{aligned}
& X_{\mathrm{sp}}^{+}(x, \lambda)=\mathbb{1}+\frac{1}{2 \pi \mathrm{i}} \int_{-\infty}^{\infty} \frac{d \mu}{\mu-\lambda} X_{\mathrm{sp}}^{-}(x, \mu) K_{1}(x, \mu)+\sum_{j=1}^{N} \frac{X_{j, \mathrm{sp}}^{-}(x) K_{1, j}(x)}{\lambda_{j}^{-}-\lambda}, \\
& X_{\mathrm{sp}}^{-}(x, \lambda)=\mathbb{1}+\frac{1}{2 \pi \mathrm{i}} \int_{-\infty}^{\infty} \frac{d \mu}{\mu-\lambda} X_{\mathrm{sp}}^{-}(x, \mu) K_{2}(x, \mu)-\sum_{j=1}^{N} \frac{X_{j, \mathrm{sp}}^{+}(x) K_{2, j}(x)}{\lambda_{j}^{+}-\lambda},
\end{aligned}
$$

where $X_{j, \mathrm{sp}}^{ \pm}(x)=X_{\mathrm{sp}}^{ \pm}\left(x, \lambda_{j}^{ \pm}\right)$and

$$
K_{1, j}(x)=\mathrm{e}^{-\mathrm{i} \lambda_{j}^{-} J x}\left(\begin{array}{cc}
0 & \rho_{j}^{+} \\
\tau_{j}^{-} & 0
\end{array}\right) \mathrm{e}^{\mathrm{i} \lambda_{j}^{-} J x}, \quad K_{2, j}(x)=\mathrm{e}^{-\mathrm{i} \lambda_{j}^{+} J x}\left(\begin{array}{cc}
0 & \tau_{j}^{+} \\
\rho_{j}^{-} & 0
\end{array}\right) \mathrm{e}^{\mathrm{i} \lambda_{j}^{+} J x} .
$$


Equations (5.9), (5.10) can be viewed as a set of singular integral equations which are equivalent to the RHP. For the MNLS these were first derived in [40].

Finally, the potential $Q(x, t)$ can be recovered from the solutions $X_{\mathrm{sp}}^{ \pm}(x, \lambda)$ of $\operatorname{RHP}(5.8)$ with a canonical normalisation (5.7). Skipping the details, we provide here only the final result:

$$
\left.Q_{\mathrm{sp}}(x, t)=\lim _{\lambda \rightarrow \infty} \lambda\left(J-X_{\mathrm{sp}}^{ \pm}(x, \lambda) J \hat{X}_{\mathrm{sp}}^{ \pm}(x, \lambda)\right]\right)=\left[J, X_{1}(x)\right],
$$

where $X_{1}(x)=\lim _{\lambda \rightarrow \infty}\left(X_{\mathrm{sp}}(x, \lambda)-\mathbb{1}\right)$.

\subsection{Definition and properties of $R_{\mathrm{sp}}^{ \pm}(x, y, \lambda)$}

The resolvent $R_{\mathrm{sp}}(\lambda)$ of $L_{\mathrm{sp}}$ is again expressed through the FAS in the form:

$$
R_{\mathrm{sp}}(\lambda) f(x)=\int_{-\infty}^{\infty} R_{\mathrm{sp}}(x, y, \lambda) f(y)
$$

where $R_{\mathrm{sp}}(x, y, \lambda)$ are given by:

$$
R_{\mathrm{sp}}(x, y, \lambda)= \begin{cases}R_{\mathrm{sp}}^{+}(x, y, \lambda) & \text { for } \lambda \in \mathbb{C}^{+}, \\ R_{\mathrm{sp}}^{-}(x, y, \lambda) & \text { for } \lambda \in \mathbb{C}^{-},\end{cases}
$$

and

$$
R_{\mathrm{sp}}^{ \pm}(x, y, \lambda)= \pm i \chi_{\mathrm{sp}}^{ \pm}(x, \lambda) \Theta^{ \pm}(x-y) \hat{\chi}_{\mathrm{sp}}^{ \pm}(y, \lambda), \quad \Theta^{ \pm}(z)=\left(\begin{array}{cc}
\theta(\mp z) \mathbb{1} & 0 \\
0 & -\theta( \pm z) \mathbb{1}
\end{array}\right) .
$$

\subsection{The dressing factors in the spinor representation}

The asymptotics of the dressing factor for $x \rightarrow \pm \infty$ are:

$$
u_{ \pm}=\exp \left(\ln c_{1}(\lambda) J_{\mathrm{sp}}\right)
$$

where

$$
u_{+}=\left(\begin{array}{cc}
\sqrt{c_{1}} \mathbb{1}_{2^{r}} & 0 \\
0 & 1 / \sqrt{c_{1}} \mathbb{1}_{2^{r-1}}
\end{array}\right), \quad u_{-}=\left(\begin{array}{cc}
1 / \sqrt{c_{1}} \mathbb{1}_{2^{r-1}} & 0 \\
0 & \sqrt{c_{1}} \mathbb{1}_{2^{r-1}}
\end{array}\right) .
$$

Thus the asymptotics of the projectors $P$ and $\bar{P}$ in the spinor representation are projectors of rank $2^{r-1}$. Since the dynamics of the MNLS does not change the rank of the projectors we conclude that the dressing factor in the spinor representation must be of the form:

$$
u(x, \lambda)=\exp \left(\frac{1}{2} \ln c_{1}(\lambda)\left(P_{1}-\bar{P}_{1}\right)\right)=\sqrt{c_{1}(\lambda)} P_{1}(x, t)+\frac{1}{\sqrt{c_{1}(\lambda)}} \bar{P}_{1}(x, t)
$$

dressing factors of such form with non-rational dependence on $\lambda$ were considered for the first time in by Ivanov [35] for the MNLS related to symplectic algebras. Here we see that such construction can be used also for the orthogonal algebras.

However when it comes to analyze the relations between the 'naked' and the dressed scattering matrices and their Gauss factors we get integer powers of $c_{1}(\lambda)$. Indeed, for the simplest case when the dressing procedure is applied just once we have:

$$
\begin{array}{ll}
T_{\mathrm{sp}}(\lambda)=\hat{u}_{+} T_{0, \mathrm{sp}}(\lambda) u_{-}(\lambda), & D_{\mathrm{sp}}^{ \pm}(\lambda)=\hat{u}_{+} D_{0, \mathrm{sp}}^{ \pm}(\lambda) u_{-}(\lambda), \\
T_{\mathrm{sp}}^{ \pm}(\lambda)=\hat{u}_{+} T_{0, \mathrm{sp}}^{ \pm}(\lambda) u_{+}(\lambda), & S_{\mathrm{sp}}^{ \pm}(\lambda)=\hat{u}_{-} S_{0, \mathrm{sp}}^{ \pm}(\lambda) u_{-}(\lambda),
\end{array}
$$


and as a consequence

$$
\begin{array}{ll}
\boldsymbol{a}_{\mathrm{sp}}^{+}(\lambda)=\frac{1}{c_{1}^{+}(\lambda)} \boldsymbol{a}_{0, \mathrm{sp}}^{+}(\lambda), & \boldsymbol{a}_{\mathrm{sp}}^{-}(\lambda)=c_{1}^{+}(\lambda) \boldsymbol{a}_{0, \mathrm{sp}}^{-}(\lambda), \\
\rho_{\mathrm{sp}}^{+}(\lambda)=\frac{1}{c_{1}^{+}(\lambda)} \rho_{0, \mathrm{sp}}^{+}(\lambda), & \rho_{\mathrm{sp}}^{-}(\lambda)=c_{1}^{+}(\lambda) \rho_{0, \mathrm{sp}}^{-}(\lambda), \\
\tau_{\mathrm{sp}}^{+}(\lambda)=\frac{1}{c_{1}^{+}(\lambda)} \tau_{0, \mathrm{sp}}^{+}(\lambda), & \tau_{\mathrm{sp}}^{-}(\lambda)=c_{1}^{+}(\lambda) \tau_{0, \mathrm{sp}}^{-}(\lambda) .
\end{array}
$$

\subsection{The spectral decompositions of $L_{\mathrm{sp}}$}

Again apply the contour integration method to the kernel $R_{\mathrm{sp}}^{ \pm}$and derive the following completeness relation:

$$
\begin{aligned}
\delta(x-y) \mathbb{1}_{2^{r}}= & \frac{1}{2 \pi} \int_{-\infty}^{\infty} d \lambda\left\{\left|\phi^{+}(x, \lambda)\right\rangle \hat{\boldsymbol{a}}^{+}(\lambda)\left\langle\psi^{+}(y, \lambda)|-| \phi^{-}(x, \lambda)\right\rangle \hat{\boldsymbol{a}}^{-}(\lambda)\left\langle\psi^{-}(y, \lambda)\right|\right\} \\
& +\sum_{j=1}^{N}\left(\operatorname{Res}_{\lambda=\lambda_{j}^{+}} R^{+}(x, y)+\operatorname{Res}_{\lambda=\lambda_{j}^{-}} R^{-}(x, y)\right) .
\end{aligned}
$$

The residues in (5.11) can be expressed by the properly normalized eigenfunctions of $L_{\mathrm{sp}}$ corresponding to the eigenvalues $\lambda_{j}^{ \pm}$.

Thus we conclude that the continuous spectrum of $L_{\mathrm{sp}}$ fills up $\mathbb{R}$ and has multiplicity $2^{r}$. The discrete eigenvalues $\lambda_{k}^{ \pm}$are simple poles of the resolvent.

\section{Dressing factors and higher representations of $\mathfrak{g}$}

Here we will briefly outline how, starting from equation (2.13) one can construct the dressing factors in any irreducible representation of the Lie algebra $\mathfrak{g}$. We will illustrate this on one of the simple nontrivial examples of $u(x, \lambda)$ with rank-1 projectors $P(x)$ and $\bar{P}(x)$. Our intention is to outline the explicit $\lambda$-dependence of $u(x, \lambda)$ in any IRREP. To this end it will be most convenient to use the first line of equation (3.1):

$$
u(x, \lambda)=\exp \left(\ln c_{1}(\lambda)\left(P_{1}-\bar{P}_{1}\right)\right) .
$$

Note that $P(x)-\bar{P}(x) \in \mathfrak{g}$ and therefore the right hand side of (6.1) will be an a Lie group element. In what follows we will assume that $\operatorname{rank} P(x)=\operatorname{rank} \bar{P}(x)=1$ and will derive explicitly the $\lambda$-dependence of the right hand side of equation (6.1) in any irreducible representation of $\mathfrak{g}$.

In fact it will be enough to analyze the $\lambda$-dependence of the asymptotic of $u(x, \lambda)$ for $x \rightarrow \pm \infty$.

$$
\lim _{x \rightarrow \infty}(P(x)-\bar{P}(x))=H_{e_{1}} .
$$

In order to be more specific we will do our considerations for the case when $\mathfrak{f} \simeq s o(7)$. This algebra is of rank 3 . We also choose the representation with highest weight

$$
\omega=3 \omega_{3}=\frac{3}{2}\left(e_{1}+e_{2}+e_{3}\right) .
$$

The structure of the weight system $\Gamma^{(\omega)}$ is described in Table 1.

It is natural to expect that $u(x, \lambda)$ will have the same type of $\lambda$-dependence [15] as its asymptotic for $x \rightarrow \pm \infty$. Therefore we will evaluate the right hand side of equation (6.1) for $x \rightarrow \infty$. Doing this we well use the known formula

$$
H_{e_{1}}=\sum_{\gamma \in \Gamma^{\left(3 \omega_{3}\right)}}\left(e_{1}, \gamma\right)|\gamma\rangle\langle\gamma| \text {. }
$$


Table 1. The structure of the weight system $\Gamma^{\left(3 \omega_{1}\right)}$ for the algebra $s o(7)$ with dimension 112 . We list here the number of weights of different lengths $\ell(\gamma)$ and their multiplicity $\mu(\gamma)$ and length. The indices $i, j, k$ are different and take values 1,2 and 3 .

\begin{tabular}{|c||c|c|c|c|}
\hline weight type & $\Gamma_{1}$ & $\Gamma_{2}$ & $\Gamma_{3}$ & $\Gamma_{4}$ \\
\hline & $\frac{3}{2}\left( \pm e_{1} \pm e_{2} \pm e_{3}\right)$ & $\frac{1}{2}\left( \pm 3 e_{i} \pm 3 e_{j} \pm e_{k}\right)$ & $\frac{1}{2}\left( \pm 3 e_{i} \pm e_{j} \pm e_{k}\right)$ & $\frac{1}{2}\left( \pm e_{1} \pm e_{2} \pm e_{3}\right)$ \\
\hline$\#(\gamma)$ & 8 & 24 & 24 & 24 \\
$\mu(\gamma)$ & 1 & 1 & 2 & 4 \\
$\ell(\gamma)$ & $\frac{27}{4}$ & $\frac{19}{4}$ & $\frac{11}{4}$ & $\frac{3}{4}$ \\
\hline
\end{tabular}

Therefore we have to arrange the weights in $\Gamma^{\left(3 \omega_{3}\right)}$ according to their scalar products with $e_{1}$, namely:

$$
\begin{aligned}
& \Gamma^{\left(3 \omega_{3}\right)}=\Gamma_{3 / 2} \cup \Gamma_{1 / 2} \cup \Gamma_{-1 / 2} \cup \Gamma_{-3 / 2}, \\
& \Gamma_{3 / 2} \equiv\left\{\frac{3}{2}\left(e_{1} \pm e_{2} \pm e_{3}\right)\right\} \cup\left\{\frac{1}{2}\left(3 e_{1} \pm 3 e_{j} \pm e_{k}\right)\right\} \cup\left\{\frac{1}{2}\left(3 e_{1} \pm e_{2} \pm e_{3}\right)\right\}, \\
& \Gamma_{1 / 2} \equiv\left\{\frac{1}{2}\left(e_{1} \pm 3 e_{2} \pm 3 e_{3}\right)\right\} \cup\left\{\frac{1}{2}\left(e_{1} \pm 3 e_{j} \pm e_{k}\right)\right\} \cup\left\{\frac{1}{2}\left(e_{1} \pm e_{2} \pm e_{3}\right)\right\}, \\
& \Gamma_{-1 / 2} \equiv\left\{\frac{1}{2}\left(-e_{1} \pm 3 e_{2} \pm 3 e_{3}\right)\right\} \cup\left\{\frac{1}{2}\left(-e_{1} \pm 3 e_{j} \pm e_{k}\right)\right\} \cup\left\{\frac{1}{2}\left(-e_{1} \pm e_{2} \pm e_{3}\right)\right\}, \\
& \Gamma_{-3 / 2} \equiv\left\{\frac{3}{2}\left(-e_{1} \pm e_{2} \pm e_{3}\right)\right\} \cup\left\{\frac{1}{2}\left(-3 e_{1} \pm 3 e_{j} \pm e_{k}\right)\right\} \cup\left\{\frac{1}{2}\left(-3 e_{1} \pm e_{2} \pm e_{3}\right)\right\},
\end{aligned}
$$

where $j, k$ take the values 2 and 3 . As a result we obtain

$$
H_{e_{1}}=\frac{3}{2} \pi_{3 / 2}+\frac{1}{2} \pi_{1 / 2}-\frac{1}{2} \pi_{-1 / 2}-\frac{3}{2} \pi_{-3 / 2},
$$

where the projectors $\pi_{a}, a= \pm \frac{3}{2}, \pm \frac{1}{2}$ are equal to

$$
\pi_{a}=\sum_{\gamma \in \Gamma_{a}}|\gamma\rangle\langle\gamma|
$$

and obviously satisfy the relations:

$$
\pi_{a} \pi_{b}=\delta_{a b} \pi_{a}, \quad \operatorname{rank} \pi_{3 / 2}=\operatorname{rank} \pi_{-3 / 2}=20, \quad \operatorname{rank} \pi_{1 / 2}=\operatorname{rank} \pi_{-1 / 2}=36,
$$

and $\pi_{3 / 2}+\pi_{1 / 2}+\pi_{-1 / 2}+\pi_{-3 / 2}=\mathbb{1}_{112}$. Thus the $Q(x)-\lambda J$ acquires the following block-matrix form:

$$
\left(\begin{array}{cccc}
\frac{3}{2} \lambda \mathbb{1}_{20} & Q_{(12)} & Q_{(13)} & Q_{(14)} \\
Q_{(21)} & \frac{1}{2} \lambda \mathbb{1}_{36} & Q_{(23)} & Q_{(24)} \\
Q_{(31)} & Q_{(32)} & -\frac{1}{2} \lambda \mathbb{1}_{36} & Q_{(34)} \\
Q_{(41)} & Q_{(42)} & Q_{(43)} & -\frac{3}{2} \lambda \mathbb{1}_{20}
\end{array}\right)
$$

Formally this potential can be viewed as related to the homogeneous space $S O(112) / S(O(40) \otimes$ $O(72)$ ). However all matrix elements of the potential $Q(x)$ are determined by the five components of the vector $\vec{q}$ and their complex conjugate. This deep reduction imposed on $Q(x)$ corresponds to the fact that instead of considering a generic element of this homogeneous space, we rather pick up the representation of $s o(7)$ with highest weight $3 \omega_{3}$. 
Inserting equation (6.2) into equation (6.1) we get:

$$
\lim _{x \rightarrow \infty} u^{\left(3 \omega_{3}\right)}(x, \lambda)=(c(\lambda))^{3 / 2} \pi_{3 / 2}+(c(\lambda))^{1 / 2} \pi_{1 / 2}+(c(\lambda))^{-1 / 2} \pi_{-1 / 2}+(c(\lambda))^{-3 / 2} \pi_{-3 / 2},
$$

and as a result for the $\lambda$-dependence of $u^{\left(3 \omega_{3}\right)}(x, \lambda)$ and its inverse we get:

$$
\begin{aligned}
& u^{\left(3 \omega_{3}\right)}(x, \lambda)=(c(\lambda))^{3 / 2} \pi_{3 / 2}(x)+(c(\lambda))^{1 / 2} \pi_{1 / 2}(x) \\
& +(c(\lambda))^{-1 / 2} \pi_{-1 / 2}(x)+(c(\lambda))^{-3 / 2} \pi_{-3 / 2}(x) \\
& \left(u^{\left(3 \omega_{3}\right)}\right)^{-1}(x, \lambda)=(c(\lambda))^{-3 / 2} \pi_{3 / 2}(x)+(c(\lambda))^{-1 / 2} \pi_{1 / 2}(x) \\
& +(c(\lambda))^{1 / 2} \pi_{-1 / 2}(x)+(c(\lambda))^{3 / 2} \pi_{-3 / 2}(x) .
\end{aligned}
$$

The four projectors $\pi_{a}(x)$ have the same properties as their asymptotic values:

$$
\begin{aligned}
& \pi_{a}(x) \pi_{b}(x)=\delta_{a b} \pi_{a}(x), \\
& \operatorname{rank} \pi_{3 / 2}(x)=\operatorname{rank} \pi_{-3 / 2}(x)=20, \quad \operatorname{rank} \pi_{1 / 2}(x)=\operatorname{rank} \pi_{-1 / 2}(x)=36 .
\end{aligned}
$$

Their explicit $x$ dependence as well as the interrelation between the potentials $Q_{(0)}(x)$ and $Q_{(1)}(x)$ follow from the equation for $u(x, \lambda)(2.12)$ considered in the representation $V^{\left(3 \omega_{3}\right)}$. In particular we get:

$$
\begin{aligned}
Q_{(1)}(x)-Q_{(0)}(x) & =\lim _{\lambda \rightarrow \infty} \lambda\left(J-u^{\left(3 \omega_{3}\right)} J\left(u^{\left(3 \omega_{3}\right)}\right)^{-1}(x, \lambda)\right) \\
& =\left(\lambda^{+}-\lambda^{-}\right)\left[J, \frac{3}{2}\left(\pi_{3 / 2}(x)-\pi_{-3 / 2}(x)\right)+\frac{1}{2}\left(\pi_{1 / 2}(x)-\pi_{-1 / 2}(x)\right)\right] .
\end{aligned}
$$

Though the expressions for the dressing factor in this representation seem to be rather complex, nevertheless they are determined uniquely by the projector $P_{1}(x)$, or equivalently, through the polarization vector $\left|n_{1}(x)\right\rangle$. The corresponding expressions can be using the fact that $V^{\left(3 \omega_{3}\right)}$ can be extracted as the invariant subspace of the tensor product $V^{\left(\omega_{3}\right)} \otimes V^{\left(\omega_{3}\right)} \otimes V^{\left(\omega_{3}\right)}$ corresponding to the highest weight vector $3 \omega_{3}$. Therefore one can conclude that the matrix elements of the projectors $\pi_{a}, a= \pm 3 / 2, \pm 1 / 2$ will be polynomials of sixth order of the components of $\left|n_{1}(x)\right\rangle$.

Our final remark concerns the analyticity properties of the FAS dressed by $u^{\left(3 \omega_{3}\right)}(x, \lambda)$. The factor itself contains powers of root square of $c(\lambda)$ which in general may lead to essential singularities at $\lambda=\lambda^{+}$and $\lambda=\lambda^{-}$. Note however that the dressed FAS in this representation are obtained from the regular solutions via the analog of equation (2.11):

$$
\begin{aligned}
& \chi_{1}^{ \pm, 3 \omega_{3}}(x, t, \lambda)=u^{\left(3 \omega_{3}\right)}(x, \lambda) \chi_{0}^{ \pm, 3 \omega_{3}}(x, t, \lambda)\left(u_{-}^{3 \omega_{3}}\right)^{-1}(\lambda), \\
& \begin{aligned}
u_{-}^{3 \omega_{3}}(\lambda) & =\lim _{x \rightarrow-\infty} u^{3 \omega_{3}}(x, \lambda)=\exp \left(-\ln c_{1}(\lambda) H_{e_{1}}\right) \\
\quad & (c(\lambda))^{3 / 2} \pi_{=3 / 2}+(c(\lambda))^{1 / 2} \pi_{-1 / 2}+(c(\lambda))^{-1 / 2} \pi_{1 / 2}+(c(\lambda))^{-3 / 2} \pi_{3 / 2} .
\end{aligned}
\end{aligned}
$$

It is not difficult to check that the right hand side of first line in equation (6.3) does not contain square root terms of $c(\lambda)$; all half-integer powers of $c(\lambda)$ get multiplied by other half-integer powers and the result is that $\chi_{1}^{ \pm, 3 \omega_{3}}(x, t, \lambda)$ acquires additional pole singularities at $\lambda=\lambda^{+}$and $\lambda=\lambda^{-}$.

\section{Conclusions}

We have analyzed the spectral properties of the Lax operators related to three different representations of $\mathfrak{g} \simeq B_{r}$ : the typical, the adjoint and the spinor representation. In all these cases the spectral properties such as: i) the multiplicity of the continuous spectra and of the discrete 
eigenvalues; ii) the explicit form of the dressing factors; iii) the completeness relations of the eigenfunctions are substantially different. However the minimal sets of scattering data $\mathfrak{T}_{i}$ are provided by the same sets of functions, i.e. the sets $\mathfrak{T}_{i}$ are invariant with respect to the choice of the representation of $\mathfrak{g}$.

Our considerations were performed for the class of smooth potentials $Q(x)$ vanishing fast enough for $x \rightarrow \pm \infty$. Similar results can be derived also for the class of potentials tending to constants $Q_{ \pm}$for $x \rightarrow \pm \infty$.

\section{A The adjoint representations of $s o(5)$ and $s o(7)$}

The adjoint representations of all orthogonal algebras $s o(2 r+1)$ and $s o(2 r)$ are characterized by the fundamental weight $\omega_{2}=e_{1}+e_{2}$. By definition the corresponding weight system is

$$
\Gamma_{\omega_{2}} \equiv \Delta \cup\{0\}_{r}
$$

where $\Delta$ is the root system of the algebra and $\{0\}$ is a vanishing weight with multiplicity $r$. We will order the weights in $\Gamma_{\omega_{2}}$ according to their scalar products with $e_{1}$.

Let us consider in more detail the two special cases of $s o(2 r+1)$ with $r=2$ and $r=3$. For $r=2$ the adjoint representation is 10 -dimensional. Ordering the roots as mentioned above we get:

$$
\Delta_{1}^{+} \simeq\left\{e_{1}+e_{2}, e_{1}, e_{1}-e_{2}\right\}, \quad \Delta_{0} \simeq\left\{e_{2},-e_{2}\right\}, \quad \Delta_{1}^{-} \simeq\left\{-e_{1}+e_{2}, e_{1},-e_{1}-e_{2}\right\},
$$

As a result the element $J$ in the adjoint representation takes the form:

$$
J_{\mathrm{ad}}=\left(\begin{array}{ccc}
\mathbb{1}_{2} & 0 & 0 \\
0 & 0 & 0 \\
0 & 0 & -\mathbb{1}_{2}
\end{array}\right), \quad Q_{\mathrm{ad}}=\left(\begin{array}{ccc}
0 & Q_{\mathrm{ad} ; 12} & 0 \\
Q_{\mathrm{ad} ; 21} & 0_{6} & Q_{\mathrm{ad} ; 23} \\
0 & Q_{\mathrm{ad} ; 32} & 0
\end{array}\right)
$$

Analogously for $r=3$ the adjoint representation is 21-dimensional. Ordering the roots as mentioned above we get:

$$
\begin{aligned}
& \Delta_{1}^{+} \simeq\left\{e_{1}+e_{2}, e_{1}+e_{3}, e_{1}, e_{1}-e_{3}, e_{1}-e_{2}\right\}, \quad \Delta_{0} \simeq\left\{e_{2} \pm e_{3},-\left(e_{2} \pm e_{3}\right)\right\}, \\
& \Delta_{1}^{-} \simeq\left\{-e_{1}+e_{2},-e_{1}+e_{3}, e_{1},-e_{1}-e_{3},-e_{1}-e_{2}\right\} .
\end{aligned}
$$

As a result the element $J$ in the adjoint representation takes the form:

$$
J_{\mathrm{ad}}=\left(\begin{array}{ccc}
\mathbb{1}_{5} & 0 & 0 \\
0 & 0 & 0 \\
0 & 0 & -\mathbb{1}_{5}
\end{array}\right), \quad Q_{\mathrm{ad}}=\left(\begin{array}{ccc}
0 & Q_{\mathrm{ad} ; 12} & 0 \\
Q_{\mathrm{ad} ; 21} & 0_{11} & Q_{\mathrm{ad} ; 23} \\
0 & Q_{\mathrm{ad} ; 32} & 0
\end{array}\right)
$$

It is not difficult to write down the explicit form of $Q_{\mathrm{ad}}$ but it will not be necessary. We have used above a more compact realization of $Q_{\mathrm{ad}} \operatorname{as~}_{\operatorname{ad}}$.

\section{B The spinor representation of $s o(5)$}

The highest weight and the weight system of $s o(5)$ are given by $[5,33]$. It is well known that $s o(5) \simeq s p(4)$ so the spinor representation of $s o(5)$ is realized through symplectic $s p(4)$ matrices

$$
\omega_{2} \equiv \gamma_{1}=\frac{1}{2}\left(e_{1}+e_{2}\right), \quad \gamma_{2}=\frac{1}{2}\left(e_{1}-e_{2}\right), \quad \gamma_{3}=-\gamma_{2}, \quad \gamma_{4}=-\gamma_{1} .
$$


The Cartan-Weyl basis of so(5) is given by

$$
\begin{array}{ll}
E_{e_{1}-e_{2}}=\Gamma_{2, \overline{2}}=\mathcal{E}_{2 \epsilon_{2}}, & E_{e_{1}+e_{2}}=\Gamma_{1, \overline{1}}=\mathcal{E}_{2 \epsilon_{1}}, \\
E_{e_{1}}=\Gamma_{1, \overline{2}}-\Gamma_{2, \overline{1}}=\mathcal{E}_{\epsilon_{1}+\epsilon_{2}}, & E_{e_{2}}=\Gamma_{1,2}-\Gamma_{2, \overline{1}}=\mathcal{E}_{\epsilon_{1}-\epsilon_{2}}, \\
H_{e_{1}}=\frac{1}{2}\left(\Gamma_{1,1}+\Gamma_{2,2}\right), & H_{e_{2}}=\frac{1}{2}\left(\Gamma_{1,1}-\Gamma_{2,2}\right),
\end{array}
$$

where $\bar{k}=5-k$ and

$$
\Gamma_{k, p}=\left|\gamma_{k}\right\rangle\left\langle\gamma_{p}\right|, \quad 1 \leq k \leq p \leq 4
$$

By $\mathcal{E}_{\epsilon_{i} \pm \epsilon_{j}}$ above we have denoted the Weyl generators of $s p(4)$. The Lax operator $L$ in the spinor representation of $s o(5)$ takes the form:

$$
L_{\mathrm{sp}} \psi_{\mathrm{sp}}=i \frac{\partial \psi_{\mathrm{sp}}}{\partial x}+\left(Q_{\mathrm{sp}}-\lambda J_{\mathrm{sp}}\right) \psi_{\mathrm{sp}}(x, \lambda)=0,
$$

where $Q_{\mathrm{sp}}(x, t)$ and $J_{\mathrm{sp}}$ are $4 \times 4$ symplectic matrices of the form:

$$
Q_{\mathrm{sp}}=\left(\begin{array}{cc}
0 & \boldsymbol{q} \\
\boldsymbol{q}^{\dagger} & 0
\end{array}\right), \quad J_{\mathrm{sp}}=\frac{1}{2}\left(\begin{array}{cc}
\mathbb{1}_{2} & 0 \\
0 & -\mathbb{1}_{2}
\end{array}\right), \quad \boldsymbol{q}(x, t)=\left(\begin{array}{cc}
q_{0} & q_{\overline{1}} \\
q_{1} & -q_{0}
\end{array}\right) .
$$

\section{The spinor representation of $s o(7)$}

The highest weight and the weight system of $s o(7)$ are given by $[5,33]$

$$
\begin{aligned}
\omega_{3} & \equiv \gamma_{1}=\frac{1}{2}\left(e_{1}+e_{2}+e_{3}\right), & \gamma_{2} & =\frac{1}{2}\left(e_{1}+e_{2}-e_{3}\right), \\
\gamma_{3} & =\frac{1}{2}\left(e_{1}-e_{2}+e_{3}\right), & \gamma_{4} & =\frac{1}{2}\left(e_{1}-e_{2}-e_{3}\right), \\
\gamma_{5} & =-\gamma_{4}, \quad \gamma_{6}=-\gamma_{3}, & \gamma_{7} & =-\gamma_{2}, \quad \gamma_{8}=-\gamma_{1} .
\end{aligned}
$$

The Cartan-Weyl basis of so(7) is given by

$$
\begin{array}{ll}
E_{e_{1}-e_{2}}=\Gamma_{3, \overline{4}}=\mathcal{E}_{\epsilon_{3}+\epsilon_{4}}, & E_{e_{2}-e_{3}}=\Gamma_{2,3}=\varepsilon_{\epsilon_{2}-\epsilon_{3}}, \\
E_{e_{1}-e_{3}}=\Gamma_{2, \overline{4}}=\mathcal{E}_{\epsilon_{2}+\epsilon_{4}}, & E_{e_{1}+e_{2}}=\Gamma_{1, \overline{2}}=\mathcal{E}_{\epsilon_{2}+\epsilon_{4}}, \\
E_{e_{1}+e_{3}}=\Gamma_{1, \overline{3}}=\mathcal{E}_{\epsilon_{1}+\epsilon_{3}}, & E_{e_{2}+e_{3}}=\Gamma_{1,4}=\mathcal{E}_{\epsilon_{1}-\epsilon_{4}}, \\
E_{e_{1}}=\Gamma_{1, \overline{4}}+\Gamma_{2, \overline{3}}=\mathcal{E}_{\epsilon_{1}+\epsilon_{4}}+\mathcal{E}_{\epsilon_{2}+\epsilon_{3}}, & E_{e_{2}}=\Gamma_{1,3}+\Gamma_{2,4}=\mathcal{E}_{\epsilon_{1}-\epsilon_{4}}+\mathcal{E}_{\epsilon_{2}-\epsilon_{4}}, \\
E_{e_{3}}=\Gamma_{1,2}-\Gamma_{3,4}=\mathcal{E}_{\epsilon_{1}-\epsilon_{2}}-\mathcal{E}_{\epsilon_{3}-\epsilon_{4}}, & H_{e_{1}}=\frac{1}{2}\left(\Gamma_{1,1}+\Gamma_{2,2}+\Gamma_{3,3}+\Gamma_{4,4}\right), \\
H_{e_{1}}=\frac{1}{2}\left(\Gamma_{1,1}+\Gamma_{2,2}-\Gamma_{3,3}-\Gamma_{4,4}\right), & H_{e_{1}}=\frac{1}{2}\left(\Gamma_{1,1}-\Gamma_{2,2}+\Gamma_{3,3}-\Gamma_{4,4}\right),
\end{array}
$$

where $\bar{k}=9-k$ and

$$
\begin{array}{ll}
\Gamma_{k, p}=\left|\gamma_{k}\right\rangle\left\langle\gamma_{p}\left|-(-1)^{k+p}\right| \gamma_{\bar{p}}\right\rangle\left\langle\gamma_{\bar{k}}\right|, & 1 \leq k \leq p \leq 4, \\
\Gamma_{k, \bar{p}}=\left|\gamma_{k}\right\rangle\left\langle\gamma_{p}\left|+(-1)^{k+p}\right| \gamma_{\bar{p}}\right\rangle\left\langle\gamma_{\bar{k}}\right|, & 1 \leq k \leq p \leq 4
\end{array}
$$

Note that the typical representation of $s o(8)$ is also 8-dimensional. So by $\mathcal{E}_{\epsilon_{i} \pm \epsilon_{j}}$ above we have denoted the Weyl generators of $s o(8)$. So we can also consider the spinor representation of $s o(7)$ as an embedding of $s o(7)$ into $s o(8)$. Such embedding can be realized by taking the average of the Cartan-Weyl basis of so(8) with respect to the $\mathbb{Z}_{2}$ external automorphism of so(8) (the mirror 
reflection) which changes $\epsilon_{4} \leftrightarrow-\epsilon_{4}$. In short the Lax operator $L$ in the spinor representation of so(7) take the form:

$$
L_{\mathrm{sp}} \psi_{\mathrm{sp}}=i \frac{\partial \psi_{\mathrm{sp}}}{\partial x}+\left(Q_{\mathrm{sp}}-\lambda J_{\mathrm{sp}}\right) \psi_{\mathrm{sp}}(x, \lambda)=0,
$$

where $Q_{\mathrm{sp}}(x, t)$ and $J_{\mathrm{sp}}$ are $8 \times 8$ matrices of the form:

$$
Q_{\mathrm{sp}}=\left(\begin{array}{cc}
0 & \boldsymbol{q} \\
\boldsymbol{q}^{\dagger} & 0
\end{array}\right), \quad J_{\mathrm{sp}}=\frac{1}{2}\left(\begin{array}{cc}
\mathbb{1}_{4} & 0 \\
0 & -\mathbb{1}_{4}
\end{array}\right), \quad \boldsymbol{q}(x, t)=\left(\begin{array}{cccc}
q_{0} & q_{\overline{2}} & q_{\overline{1}} & 0 \\
q_{2} & q_{0} & 0 & -q_{\overline{1}} \\
q_{1} & 0 & -q_{0} & q_{\overline{2}} \\
0 & -q_{1} & q_{2} & -q_{0}
\end{array}\right) .
$$

Our final remark here concerns the spinor representation with highest weight $\omega=3 \omega_{3}$. Then

$$
\Gamma \simeq\left\{\frac{3}{2}\left( \pm e_{1} \pm e_{2} \pm e_{3}\right), \frac{1}{2}\left( \pm e_{1} \pm e_{2} \pm e_{3}\right)\right\}
$$

The dimension of this representation is 8 . The explicit form of the element $J$ in this representation is $J=\operatorname{diag}\left(\frac{3}{2} \mathbb{1}, \frac{1}{2} \mathbb{1},-\frac{3}{2} \mathbb{1},-\frac{1}{2} \mathbb{1}\right)$, i.e. all eigenvalues of $J$ are non-vanishing. The potential $Q$ will have block-matrix structure compatible with the one of $J$.

\section{Acknowledgements}

The authors have the pleasure to thank Prof. Adrian Constantin, Prof. Nikolay Kostov, Prof. Alexander Mikhailov and Dr. Rossen Ivanov for numerous useful discussions. Part of the work was done during authors visit at the Erwin Schrödinger International Institute for Mathematical Physics in the framework of the research programme "Recent Advances in Integrable Systems of Hydrodynamic Type" (October 2009). One of us (GGG) is grateful to the organizers of the Kyiv conference for their hospitality. This material is based upon works supported by the Science Foundation of Ireland (SFI), under Grant No. 09/RFP/MTH2144. We thank three anonymous referees for numerous useful suggestions.

\section{References}

[1] Ablowitz M.J., Kaup D.J., Newell A.C., Segur H., The inverse scattering transform - Fourier analysis for nonlinear problems, Studies in Appl. Math. 53 (1974), 249-315.

[2] Ablowitz M.J., Prinari B., Trubatch A.D. Discrete and continuous nonlinear Schrödinger systems, London Mathematical Society Lecture Note Series, Vol. 302, Cambridge University Press, Cambridge, 2004.

[3] Athorne C., Fordy A., Generalised KdV and MKDV equations associated with symmetric spaces, J. Phys. A: Math. Gen. 20 (1987), 1377-1386.

[4] Beals R., Sattinger D.H., On the complete integrability of completely integrable systems, Comm. Math. Phys. 138 (1991), 409-436.

[5] Bourbaki N., Éléments de mathématique, Actualités Scientifiques et Industrielles, no. 1364, Hermann, Paris, 1975 (in French).

[6] Calogero F., Degasperis A., Spectral transform and solitons, Vol. I, North-Holland Publishing Co., Amsterdam - New York, 1982.

[7] Calogero F., Degasperis A., Nonlinear evolution equations solvable by the inverse spectral transform. I, Nuovo Cimento B 32 (1976), 201-242.

Calogero F., Degasperis A., Nonlinear evolution equations solvable by the inverse spectral transform. II, Nuovo Cimento B 39 (1976), 1-54.

[8] Calogero F., Degasperis A., Coupled nonlinear evolution equations solvable via the inverse spectral transform and solitons that come back: the boomeron, Lett. Nuovo Cimento 16 (1976) 425-433. 
[9] Degasperis A., Solitons, boomerons, trappons, in Nonlinear Evolution Equations Solvable by the Spectral Transform (Internat. Sympos., Accad. Lincei, Rome, 1977), Editor F. Calogero, Res. Notes in Math., Vol. 26, Pitman, Boston, Mass. - London, 1978, 97-126.

[10] Drinfel'd V.G., Sokolov V.V., Lie algebras and equations of Korteweg-de Vries type, J. Sov. Math. 30 (1985), 1975-2036.

[11] Faddeev L.D., Takhtadjan L.A., Hamiltonian methods in the theory of solitons, Springer Series in Soviet Mathematics, Springer-Verlag, Berlin, 1987.

[12] Fordy A.P., Kulish P.P., Nonlinear Schrödinger equations and simple Lie algebras, Comm. Math. Phys. 89 (1983), 427-443.

[13] Gakhov F.D., Boundary value problems, Pergamon Press, Oxford, 1966.

[14] Gerdjikov V.S., Generalized Fourier transforms for the soliton equations. Gauge-covariant formulation, Inverse Problems 2 (1986), 51-74.

Gerdjikov V.S., Generating operators for the nonlinear evolution equations of soliton type related to the semisimple Lie algebras, Doctor of Sciences Thesis, JINR, Dubna, USSR, 1987 (In Russian).

[15] Gerdjikov V.S., The Zakharov-Shabat dressing method and the representation theory of the semisimple Lie algebras, Phys. Lett. A 126 (1987), 184-188.

[16] Gerdjikov V.S., Complete integrability, gauge equivalence and Lax representations of the inhomogeneous nonlinear evolution equations, Teoret. Mat. Fiz. 92 (1992), 374-386 (English transl.: Theoret. and Math. Phys. 92 (1992), 952-963).

[17] Gerdjikov V.S., Algebraic and analytic aspects of soliton type equations, in The Legacy of the Inverse Scattering Transform in Applied Mathematics (South Hadley, MA, 2001), Contemp. Math., Vol. 301, Amer. Math. Soc., Providence, RI, 2002, 35-68, nlin.SI/0206014.

[18] Gerdjikov V.S., Basic aspects of soliton theory, in Geometry, Integrability and Quantization, Editors I.M. Mladenov and A.C. Hirshfeld, Softex, Sofia, 2005, 78-125, nlin.SI/0604004.

[19] Gerdjikov V.S., On the spectral theory of the integro-differential operator $\Lambda$ generating nonlinear evolution equations, Lett. Math. Phys. 6 (1982), 315-323.

[20] Gerdjikov V.S., The generalized Zakharov-Shabat system and the soliton perturbations, Teoret. Mat. Fiz. 99 (1994), 292-299 (English transl.: Theoret. and Math. Phys. 99 (1994), 593-598).

[21] Gerdjikov V.S., Grahovski G.G., Kostov N.A., Reductions of $N$-wave interactions related to low-rank simple Lie algebras. I. $\mathbb{Z}_{2}$-reductions, J. Phys. A: Math. Gen. 34 (2001), 9425-9461, nlin.SI/0006001.

[22] Gerdjikov V.S., On spectral theory of Lax operators on symmetric spaces: vanishing versus constant boundary conditions, J. Geom. Symmetry Phys. 15 (2009), 1-41.

[23] Gerdjikov V.S., Grahovski G.G., Ivanov R.I., Kostov N.A., N-wave interactions related to simple Lie algebras. $\mathbb{Z}_{2}$-reductions and soliton solutions, Inverse Problems 17 (2001), 999-1015, nlin.SI/0009034.

[24] Gerdjikov V.S., Grahovski G.G., Kostov N.A., Multicomponent equations of the nonlinear Schrödinger type on symmetric spaces and their reductions, Teoret. Mat. Fiz. 144 (2005), 313-323 (English transl.: Theoret. and Math. Phys. 144 (2005), 1147-1156).

[25] Gerdjikov V.S., Grahovski G.G., Kostov N.A., On the multi-component NLS-type equations on symmetric spaces: reductions and soliton solutions, Talk at Sixth International Conference "Geometry, Integrability and Quantization" (July 3-10, 2004, Varna).

[26] Gerdjikov V.S., Grahovski G.G., Kostov N.A., On the multi-component NLS-type Models and their gauge equivalent, Talk at the International Conference "Contemporary Aspects of Astronomy, Theoretical and Gravitational Physics" (May 20-22, 2004, Sofia).

[27] Gerdjikov V.S., Kaup D.J., Kostov N.A., Valchev T.I., Bose-Einstein condensates and multi-component NLS models on symmetric spaces of BD.I-type. Expansions over squared solutions, in Proceedings of the Conference on Nonlinear Science and Complexity (July 28-31, 2008, Porto, Portugal), to appear.

[28] Gerdjikov V.S., Kostov N.A., Reductions of multicomponent mKdV equations on symmetric spaces of DIIItype, SIGMA 4 (2008), 029, 30 pages, arXiv:0803.1651.

[29] Gerdjikov V.S., Kostov N.A., Valchev T.I., Solutions of multi-component NLS models and spinor BoseEinstein condensates, Phys. D 238 (2009), 1306-1310, arXiv:0802.4398.

[30] Gerdjikov V.S., Kulish P.P., Expansions over the "squared" eigenfunctions of the matrix linear $n \times n$ system, Zap. Nauchn. Semin. Leningr. Otd. Mat. Inst. Steklova 101 (1981), 46-63 (in Russian).

Gerdjikov V.S., Kulish P.P., The generating operator for the $n \times n$ linear system, Phys. D 3 (1981), 549-564. 
[31] Gerdjikov V.S., Vilasi G., Yanovski A.B., Integrable Hamiltonian hierarchies. Spectral and geometric methods, Lecture Notes in Physics, Vol. 748, Springer-Verlag, Berlin, 2008.

[32] Grahovski G.G., Gerdjikov V.S., Kostov N.A., Atanasov V.A., New integrable multi-component NLS type equations on symmetric spaces: $Z_{4}$ and $Z_{6}$ reductions, in Geometry, Integrability and Quantization VII, Editors I. Mladenov and M. De Leon, Softex, Sofia, 2006, 154-175, nlin.SI/0603066.

[33] Helgasson S. Differential geometry, Lie groups and symmetric spaces, Pure and Applied Mathematics, Vol. 80, Academic Press, Inc. New York - London, 1978.

[34] Ieda J., Miyakawa T., Wadati M., Exact analysis of soliton dynamics in spinor Bose-Einstein condensates, Phys. Rev. Lett. 93 (2004), 194102, 4 pages.

[35] Ivanov R.I., On the dressing method for the generalized Zakharov-Shabat system, Nuclear Phys. B 694 (2004), 509-524, math-ph/0402031.

[36] Kaup D.J., Newell A.C., Soliton equations, singular dispersion relations and moving eigenvalues, Adv. in Math. 31 (1979), 67-100.

[37] Kostov N.A., Atanasov V.A., Gerdjikov V.S., Grahovski G.G., On the soliton solutions of the spinor BoseEinstein condensate, Proceedings of SPIE 6604 (2007), 66041T, 5 pages.

[38] Li L., Li Z., Malomed B.A., Mihalache D., Liu W.M., Exact soliton solutions and nonlinear modulation instability in spinor Bose-Einstein condensates, Phys. Rev. A 72 (2005), 033611, 11 pages, nlin.PS/0603027.

[39] Lombardo S., Mikhailov A.V., Reductions of integrable equations: dihedral group, J. Phys. A: Math. Gen. 37 (2004), 7727-7742, nlin.SI/0404013.

Lombardo S., Mikhailov A.V., Reduction groups and automorphic Lie algebras, Comm. Math. Phys. 258 (2005), 179-202, math-ph/0407048.

[40] Manakov S.V., On the theory of two-dimensional stationary self-focusing electromagnetic waves, Zh. Eksper. Teoret. Fiz. 65 (1973), 505-516 (English transl.: Sov. Phys. JETP 38 (1974) 248-253).

[41] Mikhailov A.V., The reduction problem and the inverse scattering problem, Phys. D 3 (1981), 73-117.

[42] Shabat A.B., The inverse scattering problem for a system of differential equations, Funkcional. Anal. $i$ Prilozhen. 9 (1975), no. 3, 75-78 (in Russian).

[43] Shabat A.B., An inverse scattering problem, Differentsial'nye Uravneniya 15 (1979), 1824-1834 (in Russian).

[44] Uchiyama M., Ieda J., Wadati M., Multicomponent bright solitons in $F=2$ spinor Bose-Einstein condensates, J. Phys. Soc. Japan 76 (2007), 74005, 6 pages, cond-mat/0703805.

[45] Ueda M., Koashi M., Theory of spin-2 Bose-Einstein condensates: spin correlations, magnetic response, and excitation spectra, Phys. Rev. A 65 (2002), 063602, 22 pages, cond-mat/0203052.

[46] Wadati M., The modified Korteweg-de Vries equation, J. Phys. Soc. Japan 34 (1972), 1289-1296.

[47] Zakharov V.E., Manakov S.V., Novikov S.P., Pitaevskii L.I., Theory of solitons: the inverse scattering method, Contemporary Soviet Mathematics, Consultants Bureau, New York, 1984.

[48] Zakharov V.E., Mikhailov A.V., On the integrability of classical spinor models in two-dimensional spacetime, Comm. Math. Phys. 74 (1980), 21-40.

[49] Zakharov V.E., Shabat A.B., Exact theory of two-dimensional self-focusing and one-dimensional selfmodulation of waves in nonlinear media, Zh. Eksper. Teoret. Fiz. 61 (1971), 118-134 (English transl.: Sov. Phys. JETP 34 (1972), 62-69).

[50] Zakharov V.E., Shabat A.B., A scheme for integrating nonlinear equations of mathematical physics by the method of the inverse scattering transform. I, Funkcional. Anal. i Prilozhen. 8 (1974), no. 3, 43-53 (in Russian).

Zakharov V.E., Shabat A.B., A scheme for integrating nonlinear equations of mathematical physics by the method of the inverse scattering transform. II, Funkcional. Anal. i Prilozhen. 13 (1979), no. 3, 13-23 (in Russian). 